\title{
$(\mu, \eta)$-pseudo almost automorphic solutions of a new class of competitive Lotka-Volterra model with mixed delays
}

https://doi.org/10.1515/msds-2020-0120

Received September 8, 2020; accepted December 18, 2020

\begin{abstract}
In the natural world, competition is an important phenomenon that can manifest in various generalized environments (economy, physics, ecology, biology,...). One of the famous models which is able to represent this concept is the Lotka-Volterra model. A new class of a competitive Lotka-Volterra model with mixed delays and oscillatory coefficients is investigated in this work. Thus, by using the $(\mu, \eta)$-pseudo almost automorphic functions function class and the Leray-Schauder fixed-point theorem, it can be proven that solutions exist. In addition, in such situations, we have a number of species that coexist and all the rest will be extinct. Therefore, the study of permanence becomes unavoidable. Therefore, sufficient and new conditions are given in order to establish the permanence of species without using a comparison theorem. By the new Lyapunov function we prove the asymptotic stability for the considered model. Moreover, we investigate the globally exponential stability of the $(\mu, \eta)$-pseudo almost automorphic solutions. In the end, an example is given to support theoretical result feasibility.
\end{abstract}

Keywords: Lotka-Volterra, $(\mu, \eta)$-pseudo almost automorphic, Leray-Schauder fixed point, Permanence, Stability

PACS: 34D23,34D20,47H10

\section{Introduction}

The population dynamics is a part of mathematical biology that aims to describe, in terms of mathematical models, the interaction between various types of populations in a given environment (e.g. animal populations in ecology, cell populations in biology, viral populations in epidemiology). These models are governed by evolutionary equations such as differences, functional equations, partial derivatives or stochastic equations. The interaction between populations generally takes place in a fluctuating environment over time. For example, the temperature, humidity and availability of nutrients or water are physical parameters that vary over time with the variation of seasons. In addition, there exist in population dynamics different interactions between populations (mutualism, competition, predator-prey, etc.). One of the most famous and important population dynamics model is the Lotka-Volterra ecological population model proposed by Lotka and Volterra. This model was first proposed to describe the predator-prey relationship in an ecosystem, and soon became well known and formed the basis of many important models in mathematical biology, population dynamics, biotechnology, ecology, etc. Furthermore, it describes several interacting between species. In

\footnotetext{
^Corresponding Author: Manel Amdouni: MaPSFA, ISSAT, Université de Sousse, Sousse, Tunisia, E-mail: manel.amdouni@hotmail.fr

Farouk Chérif: ISSATs and Laboratory of Mathematical Physic, Specials Functions and Applications, LR11ES35, Ecole Supérieure des Sciences et de Technologie de Hammam-Sousse, Université de Sousse, Sousse, Tunisia, E-mail: faroukcheriff@yahoo.fr
} 
these approach, the interaction of individuals of the same species is not the only one that modifies the strategy of population dynamics, but also the interaction between individuals of different species. For example, many populations may compete to exploit the resources of the environment. This is the case of competition interaction. Along the same vein, the pioneer study of Lotka and Volterra proposed an original equation class in order to model these population dynamics (see [15, 24, 28, 29]), given by

$$
z_{i}^{\prime}(t)=z_{i}(t)\left[\ell_{i}(t)-\sum_{j=1}^{n} \wp_{i j}(t) z_{j}(t)\right] ; i=1,2, \ldots, n .
$$

where $z_{i}(t)$ is the population size of species $i$ and the derivative $z_{i}^{\prime}(t)$ represent the variation of populations over time, $\ell_{i}$ is the growth rate of species $i$, and $\wp_{i j} \geq 0$ represents the effect of the population interaction.

In addition, in [10] the authors employed the above model by considering an important factor, which was the effect of toxins, and they considered the following system

$$
\begin{aligned}
& z_{1}^{\prime}(t)=z_{1}(t)\left[\ell_{1}(t)-\wp_{1}(t) z_{1}(t)-\omega_{1}(t) z_{2}(t)-\vartheta_{1}(t) z_{1}(t) z_{2}(t)\right] \\
& z_{2}^{\prime}(t)=z_{2}(t)\left[\ell_{2}(t)-\wp_{2}(t) z_{1}(t)-\omega_{2}(t) z_{2}(t)-\vartheta_{2}(t) z_{1}(t) z_{2}(t)\right]
\end{aligned}
$$

where $z_{i}(t)$ denotes the $i$-th species population density at time $t$ for the common pool of resources. Terms $\vartheta_{1} z_{1}^{2}(t) z_{2}(t)$ and $\vartheta_{2} z_{1}(t) z_{2}^{2}(t)$ denote the toxic substance effects.

Furthermore, in some specific situations, people might hope for changing the position of an existing solution, with the aim of keeping stability. This is of high importance for controlling the ecology balance. To realize this, we can alter the system structurally through the introduction of some feedback control variables with the goal of stabilizing a population at another solution. Balancing between two fish populations within one closed pond was explained by V. Volterra in 1931 using the theory of feedback. On the other hand, systems of differential equations with delay argument occupy more than a place of central importance in all areas of science and particularly in the biological sciences. These equations are used as models to describe many physical and biological systems (e.g., population dynamics and epidemiology). In fact, many actual systems have the property of aftereffect, i.e., the future states depend not only on the present, but also on the past history. Aftereffect is believed to occur in mechanics, control theory, physics, chemistry, biology, medicine, economics, atomic energy, information theory, etc. Moreover, as it is well known, in general, it is not easy to find the solutions of all linear and nonlinear differential equations. But, finding solutions becomes more difficult for delay differential equations compared to the differential equations without delay. Therefore, it is very important to determine the qualitative behavior of solutions when there is a delay. In contrast, the time delay has an influence on the dynamics of a population or several interacting species that can arise from a great variety in the system ecology, such as gestation and maturation. In [18], Gopalsamy and Weng considered the following model

$$
\begin{aligned}
& z^{\prime}(t)=\ell z(t)\left[1-\left(\frac{\wp_{1} z(t)+\wp_{2} z(t-o)}{K}\right)-q p(t)\right] \\
& p^{\prime}(t)=-\wp p(t)+\omega z(t-o) .
\end{aligned}
$$

They discussed the positive equilibrium and the globally asymptotic stability.

It should be pointed out that the most utilized concepts to solve the Lotka-Volterra system are periodic or almost periodic. Nevertheless, a lot of phenomena process some regularity, while being non periodic. It is well known that the biological system is frequently deeply disturbed by human exploring actions, such as planting and harvesting, which indicates that it is in general impossible to be explained by periodic or almost periodic situations. On the other hand, the concept of $(\mu, \eta)$-pseudo almost automorphic periodic can be in fact a natural generalization of periodicity, almost periodicity as well as almost automorphic functions. In this paper, we consider a recent $(\mu, \eta)$-pseudo almost automorphic functions concept to have a more precise description of our model. 
Moreover, in [16] the authors used the concept of almost periodicity and studied the following system

$$
\begin{aligned}
z_{i}^{\prime}(t)= & z_{i}(t)\left[\ell_{i}(t)-\sum_{j=1}^{N} \wp_{i j}(t) z_{j}^{\alpha_{i j}}(t)-\sum_{j=1}^{N} \omega_{i j}(t) z_{j}^{\beta_{i j}}\left(t-e_{i j}(t)\right)\right. \\
& \left.-q_{i}(t) p_{i}(t)-\sum_{j=1, j \neq i}^{N} v_{i j}(t) z_{i}^{\alpha_{i i}}(t) z_{j}^{\alpha_{i j}}(t)-\sum_{j=1}^{N} \int_{-\theta_{i j}}^{0} \kappa_{i j}(t, s) z_{j}^{\gamma_{i j}}(t+s) d s\right], \\
p_{i}^{\prime}(t)= & r_{i}(t)-k_{i}(t) p_{i}(t)+v_{i}(t) z_{i}^{\alpha_{i i}}(t) .
\end{aligned}
$$

where $v_{i}$ measures the controllable rate, $k_{i}$ is the control species death rate, $r_{i}$ denotes the control species input, $\alpha_{i j}, \beta_{i j}$ and $\gamma_{i j}$ provide an intra-specific interference nonlinear measure, $q_{i}$ denotes the external input rate, $v_{i j}$ is the toxic inhibition rate of the $i$-th species by others and vice versa, $\wp_{i j}$ and $\omega_{i j}$ measure the competition amount between $i$-th and $j$-th species $(i \neq j), \ell_{i}$ denotes the intrinsic exponential growth rate of the $i$-th species, $p_{i}$ is the control variable, and $z_{i}$ is the $i$-th species population density.

In biology there is a very important concept that ensures the survival of the biological species, which is the concept of permanence which implies the survival of all the species that exist initially. is introduced by Schuster, Sigmund and Wolff (1979). The notion of permanence is a mathematical tool that allows both to give a mathematical meaning to the notion of lo ng-term survival of populations in biology, and also to obtain important properties on the asymptotic behavior of solutions. Among the various investigations, the permanence or even persistence is related to the problem of coexistence species has received much attention in recent decades. Further, the notion of permanence a great interested in the study of the Lotka-Volterra system. In [16], the important problem was to study the persistence of the above system by using the comparison theorem. Afterward, they obtained the existence of a solution via the proprieties of almost periodic functions. The authors studied through an appropriate Lyapunov function, the global asymptotic stability.

The solutions of (2) will satisfy the following initial conditions

$$
\begin{aligned}
& z_{i}(s)=\xi_{i}(s) \in C\left([-e, 0], \mathbb{R}_{+}\right), \xi_{i}(0)>0 \\
& p_{i}(s)=\rho_{i}(s) \in C\left([-e, 0], \mathbb{R}_{+}\right), \rho_{i}(0)>0,
\end{aligned}
$$

with $e=\sup _{t}\left\{e_{i j}(t), \theta_{i j}, i, j=1,2, \ldots, N\right\}$.

Inspired by previous studies, our motivation of this paper consists in studying a $(\mu, \eta)$-pseudo almost automorphic solution of system (2), which has not existed up to now. One of the very serious and interesting topics in the study of differential equations is that if the force function and/ or the coefficients possesses a specific property, are we going to find the same characteristics in the solution under some delay?

Generally, compared with the previous works, the main contributions of our paper are

- Our findings generalize the results in the literature $([16,25,26])$, since the $(\mu, \eta)$-pseudo almost automorphic functions class contains classes of periodic, almost automorphic, almost periodic, pseudo almost periodic, pseudo almost automorphic and $\mu$-pseudo almost automorphic functions.

- Our conditions are different from those in ([16, 25-27]), which can be used to prove the existence, permanence and stability of other models.

- In the latter studies, the authors proved the existence of almost periodic solutions using properties related to the almost periodic solutions (see [16, 25-27]). We prove the existence of the solutions of system(2), by utilising the combination between the Leray-Schauder fixed point theorem and the exponential dichotomy, which has been never used for studying the model of Lotka-Volterra.

- Permanence is a mathematical tool that allows not only giving a mathematical meaning to the notion of long-term survival of populations in biology, but also obtainning important properties on the asymptotic behavior of solutions $([15,16,29])$. Among the various investigations, permanence or even persistence is related to the problem of coexistence species, which has received much attention in recent decades. However, the study of permanence is dedicated to use the comparison theorem ( see [11, 16, 25-27]). For this, we prove the permanence of the solutions, without using the comparison theorem.

- In this work, by new and sufficient conditions, we study such globally asymptotic stability by using a new Lyapunov function, which is completely different from that presented in ([16]). It is worth mention- 
ing that, in the work of ([16, 25-27]), the authors proved only the asymptotic or attractive stability of the almost periodic solution (not the exponential stability). Besides, we study in this paper the globally exponential stability of our model.

The paper is organized as follows: Some characteristics of the $(\mu, \eta)$-pseudo almost automorphic functions are recalled in section 2 . Addition to that, several necessary notations, definitions, and preliminaries are introduced for ulterior. In section 3, firstly, via exponential dichotomy, our system is transformed into one equation. Secondly, the permanence of the new equation is proven. In section 4, we derive conditions enough for the existence of $(\mu, \eta)$-pseudo almost automorphic solutions of the new equation within a suited convex set. In section 5 , we drive sufficient conditions in order to study the global asymptotic and exponential stabilities of the solutions. An illustrative example is presented in section 6 .

\section{Assumptions, definitions and lemmas}

Definition 1. [7] A continuous function $G: \mathbb{R} \rightarrow \mathbb{R}$ can be considered as almost automorphic if for every sequence $\left(e_{n}\right)_{n \in \mathbb{N}}$ there exists an $\left(r_{n}\right)_{n \in \mathbb{N}} \subset\left(e_{n}\right)_{n \in \mathbb{N}}$ subsequence such that

$$
\lim _{n \rightarrow \infty} G\left(t+r_{n}\right)=A(t)
$$

exists for all $t \in \mathbb{R}$, and

$$
\lim _{n \rightarrow \infty} A\left(t-r_{n}\right)=G(t) \text { for all } t \in \mathbb{R}
$$

are well defined for each $t \in \mathbb{R}$.

Let $A A(\mathbb{R}, \mathbb{R})$ basically the collection of all almost automorphic functions from $\mathbb{R}$ to $\mathbb{R}$.

Theorem 1. [6] Let $H \in A A_{U}(\mathbb{R} \times X, Y)$ ( see[8]). As a result, a superposition operator $N_{H}$ actually determined as follows:

$$
N_{H}: A A(\mathbb{R}, Y) \rightarrow A A(\mathbb{R}, Y), N_{H}(u):=[t \mapsto H(u(t), t)],
$$

is well defined continuous from $A A(\mathbb{R}, Y)$ into $A A(\mathbb{R}, Y)$.

Theorem 2. [6] Let $H \in A A_{U}(\mathbb{R} \times X, Y)$ (see[8]). If $z \in A A(\mathbb{R}, X)$, then $[t \mapsto H(t, z(t))] \in A A(\mathbb{R}, Y)$.

Let $\mathcal{D}$ be a Lebesgue $\sigma$-field of $\mathbb{R}, \mathcal{J}$ denote a set of every positive measure $\eta$ on $\mathcal{D}$ satisfying $\eta(\mathbb{R})=\infty$ and $\eta([c, d])<\infty$ for all $c, d \in \mathbb{R}(c \leq d)$.

Definition 2. [13] For $\mu, \eta \in \mathcal{J}$, the $\mu$ and $\eta$ measures can be considered as equivalent if there exist constants $c, d>0$ and a bounded interval $J \subset \mathbb{R}$ such that

$$
c \eta(Q) \leq \mu(Q) \leq d \eta(Q)
$$

for all $Q \in \mathcal{D}$ satisfying $Q \cap J=\emptyset$.

Now, the concept of ergodicity is to be introduced.

Definition 3. [13] Let $\mu, \eta \in \mathcal{J}$. A bounded continuous function $D: \mathbb{R} \rightarrow \mathbb{R}^{N}$ can be considered as $(\mu, \eta)$ ergodic if

$$
\lim _{w \rightarrow \infty} \frac{1}{\eta([-w, w])} \int_{[-w, w]}\|D(t)\| d \mu(t)=0 .
$$

The collection of all such functions is denoted by $\varepsilon\left(\mathbb{R}, \mathbb{R}^{N}, \mu, \eta\right)$. 
Definition 4. [13] Let $\mu, \eta \in \mathcal{J}$. A function $D \in B C\left(\mathbb{R}, \mathbb{R}^{N}\right)$ can be considered as $(\mu, \eta)$-pseudo almost automorphic if it is expressed by $D=M+H$, with $M \in A A\left(\mathbb{R}, \mathbb{R}^{N}\right)$ and $H \in \varepsilon\left(\mathbb{R}, \mathbb{R}^{N}, \mu, \eta\right)$. Indeed, a collection of such functions is expressed as $P A A\left(\mathbb{R}, \mathbb{R}^{N}, \mu, \eta\right)$.

Now, the next hypothesis (A1) is considered:

(i) Let $\mu, \eta \in \mathcal{J}$, with $\lim \sup _{w \rightarrow \infty} \frac{\mu([-w, w])}{\eta([-w, w])}<\infty$.

(ii) For all $w \in \mathbb{R}$ we can actually have $\epsilon>0$ besides one bounded interval $J$ in a certain way that $\mu(\{c+w$ : $c \in P\}) \leq \epsilon \mu(P)$ and this is in case $P \in \mathcal{D}$ fulfills $P \cap J=\varnothing$.

Lemma 1. [13] By (A1), we have for all $w>0$,

$$
\limsup _{s \rightarrow \infty} \frac{\mu([-s-w, s+w])}{\mu([-s, s])}<\infty .
$$

Theorem 3. [13] Let $\mu, \eta \in \mathcal{J}$, satisfy (A1)-(i). The ( $\mu, \eta)$-pseudo almost automorphic decomposition function is unique.

Throughout this paper, the following notations will be adapted, for an $H: \mathbb{R} \rightarrow \mathbb{R}_{+}$continuous function:

$$
\bar{H}=\sup _{t \in \mathbb{R}} H(t), \underline{H}=\inf _{t \in \mathbb{R}} H(t) .
$$

It is also assumed that the following conditions $(A 2)-(A 4)$ holds, where

(A2) There exists a continuous function $\lambda: \mathbb{R} \rightarrow \mathbb{R}_{+}$as follows

$$
d \mu_{\theta}(s)=\lambda(s) d \mu(s), \forall s \in \mathbb{R} .
$$

with $\mu_{\theta}(Q)=\mu\left((J-\theta)^{-1}(Q)\right)$ for all $Q \in \mathcal{D}$.

(A3) For all $1 \leq i \leq N, M\left[\ell_{i}\right]>0, M\left[k_{i}\right]>0$ and $\underline{\wp}_{i i}>0$.

(A4) $t \mapsto \kappa(t, s)$ is $(\mu, \eta)$-pseudo almost automorphic in $t \in \mathbb{R}$ uniformly with respect to $s \in\left[-\theta_{i j}, 0\right], \kappa_{i j}(t, s)$ is integrable where $s \in\left[-\theta_{i j}, 0\right]$.

\section{Permanence of the solutions}

Lemma 2. Let consider assumptions (A3) hold. For every $1 \leq i \leq N$, the following equation

$$
p_{i}^{\prime}(t)=-k_{i}(t) p_{i}(t)+v_{i}(t) z_{i}^{\alpha_{i i}}(t)+r_{i}(t)
$$

has a unique solution $\left(\Psi z_{i}\right)(t)$, given as follows

$$
\left(\Psi z_{i}\right)(t)=\int_{\infty}^{t} e^{-\int_{t}^{s} k_{i}(u) d u}\left(r_{i}(s)+v_{i}(s) z_{i}^{\alpha_{i i}}(s)\right) d s .
$$

Proof. Since for all $1 \leq i \leq N, \underline{k}_{i}>0$, then the linear equation

$$
p_{i}^{\prime}(t)=-k_{i}(t) p_{i}(t)
$$

admits on $\mathbb{R}$ an exponential dichotomy. Moreover, equation (4) has got one special formal solution

$$
\left(\Psi z_{i}\right)(t)=\int_{\infty}^{t} e^{-\int_{t}^{s} k_{i}(u) d u}\left[r_{i}(s)+v_{i}(s) z_{i}^{\alpha_{i i}}(s)\right] d s .
$$


Besides, the study of system (2) is equivalent to the following equation

$$
\begin{aligned}
z_{i}^{\prime}(t)= & z_{i}(t)\left[\ell_{i}(t)-\sum_{j=1}^{N} \wp_{i j}(t) z_{j}^{\alpha_{i j}}(t)-\sum_{j=1}^{N} \omega_{i j}(t) z_{j}^{\beta_{i j}}\left(t-e_{i j}(t)\right)\right. \\
& \left.-q_{i}(t)\left(\Psi z_{i}\right)(t)-\sum_{j=1, j \neq i}^{N} v_{i j}(t) z_{i}^{\alpha_{i i}}(t) z_{j}^{\alpha_{i j}}(t)-\sum_{j=1}^{N} \int_{-\theta_{i j}}^{0} \kappa_{i j}(t, u) z_{j}^{\gamma_{i j}}(t+u) d u\right], \\
z_{i}(s)= & \varphi_{i}(s) .
\end{aligned}
$$

Next, in order to show the existence, permanence and stability of the $(\mu, \eta)$-pseudo almost automorphic of equation (2), we need only to study the system (5).

Theorem 4. Under (A3) - (A4), there exists $J_{1}>0$ such that

$$
0 \leq z_{i}(t) \leq R_{2} \text {, for all } t \geq J_{1}, i=1, \ldots, N \text {. }
$$

Proof. Let $Z(t)=\left(z_{1}(t), \ldots, z_{N}(t)\right)$ be any positive solution of equation (5). Define $\Phi(Z(t))=\max _{1 \leq i \leq N} z_{i}(t)$. For $t>0$ provided, we have an $i(1 \leq i \leq N)$ integer in the following way

$$
\Phi(t)=\Phi(Z(t))=z_{i}(t)
$$

When we calculate an upper right $\Phi(t)$ derivative, we can get the following

$$
\begin{aligned}
D^{+} \Phi(t) & =z_{i}^{\prime}(t) \\
& \leq \Phi(t)\left[\ell_{i}(t)-\wp_{i i}(t) \Phi^{\alpha_{i i}}\right] .
\end{aligned}
$$

Denote by

$$
R_{2}=\max _{1 \leq i \leq N}\left(\frac{\bar{\ell}_{i}}{\varrho_{i i}}\right)^{\frac{1}{\alpha_{i i}}}+\left(\frac{\iota}{\varrho_{i i}}\right)^{\frac{1}{\alpha_{i i}}},
$$

with $\iota$ being any positive constant.

As a matter of fact, for any $R>0$, if $\Phi(t) \geq R_{2}$ for all $t \geq R$, then $D^{+} \Phi \leq-\iota \Phi$ for all $t \geq R$. As a result, there will be a contradiction. Hence, there should exist a $J_{1}>R$ in a certain way that $\Phi\left(J_{1}\right) \leq C$. If $\Phi(t) \leq R_{2}$, for all $t \geq J_{1}$ then $\Phi(t)$ is bounded. Otherwise, let us consider $\Phi\left(J_{2}\right) \geq R_{2}$, where $J_{2}>J_{1}$. Accordingly, there exists $J_{3}$ et $J_{4}$ such that

$$
\begin{aligned}
\Phi\left(J_{3}\right) & =\Phi\left(J_{4}\right)=R_{2}, \\
\Phi(t) & >R_{2}, \text { for all } J_{3}<t<J_{4}, \text { where } J_{1} \leq J_{3}<J_{2} \leq J_{4} .
\end{aligned}
$$

As a consequence, $\Phi(t)$ has a maximum with $J_{3} \leq t \leq J_{4}$ at least. Let consider, $\Phi(t)$ attaining its maximum at $J_{5}\left(J_{3}<J_{5}<J_{4}\right)$. Thus, $D^{+} \Phi(t)>0$, for $J_{5}-\delta<t<J_{5}$, for $\delta$ is a positive constant, which contradicts what follows

$$
D^{+} \Phi \leq \Phi(t) \max _{1 \leq i \leq N}\left[\ell_{i}(t)-\wp_{i i}(t) \Phi^{\alpha_{i i}}\right]
$$

Therefrom, there will be $\Phi(t) \leq R_{2}$ for all $t \geq J_{1}$, that is to say $z_{i}(t) \leq R_{2}$ for $t \geq J_{1}$. Let $\varepsilon \rightarrow 0$, one has $R_{2}=\max _{1 \leq i \leq N}\left(\frac{\bar{\ell}_{i}}{\underline{\wp}_{i i}}\right)^{\frac{1}{\alpha_{i i}}}$.

Lemma 3. Under (A3)-(A4), and

$$
\begin{aligned}
(A 5)\left[\ell_{i}(t)\right. & \left.\left.-\sum_{j=1}^{N}\left(\wp_{i j}(t) R_{2}^{\alpha_{i j}}+\omega_{i j}(t) R_{2}^{\beta_{i j}}+R_{2}^{\gamma_{i j}} \int_{-\theta_{i j}}^{0} \kappa_{i j}(t, s) d s\right)-\sum_{j=1, j \neq i}^{N} v_{i j}(t) R_{2}^{\alpha_{i i}} R_{2}^{\alpha_{i j}}\right)-\frac{q_{i}(t)}{\underline{k}_{i}}\left(\bar{v}_{i} R_{2}^{\alpha_{i i}}+\bar{r}_{i}\right)\right] \\
& \left.\geq\left[\underline{\ell}_{i}-\sum_{j=1}^{N}\left(\bar{\wp}_{i j} R_{2}^{\alpha_{i j}}+\bar{\omega}_{i j} R_{2}^{\beta_{i j}}+R_{2}^{\gamma_{i j}} \sup _{t} \int_{-\theta_{i j}}^{0} \kappa_{i j}(t, s) d s\right)-\frac{\bar{q}_{i}}{\underline{e}_{i}}\left(\bar{v}_{i} R_{2}^{\alpha_{i i}}+\bar{r}_{i}\right)-\sum_{j=1, j \neq i}^{N} \bar{v}_{i j} R_{2}^{\alpha_{i i}} R_{2}^{\alpha_{i j}}\right)\right]>0,
\end{aligned}
$$


there exists $R_{1}>0$ such that for all $i=1, \ldots, N$, and for all $t \geq J_{1}$, we have

$$
z_{i}(t) \geq R_{1}
$$

Proof. Define $G(t)=\min _{1 \leq i \leq N} z_{i}(t)$. For each provided $t>0$, we actually have one $i(1 \leq i \leq N)$ as follows

$$
G(t)=G(Z(t))=z_{i}(t)
$$

Next, it must be proven that $G(t) \geq R_{1}$, for $t \geq J_{1}$. By contradiction, we consider $G(t)<R_{1}$ for $t \geq-e$, for that reason

$$
\begin{aligned}
G^{\prime}(t) \geq & G(t)\left[\underline{\ell}_{i}-\sum_{j=1, j \neq i}^{N}\left(\bar{\wp}_{i j} R_{2}^{\alpha_{i j}}+\bar{v}_{i j} R_{2}^{\alpha_{i i}} R_{2}^{\alpha_{i j}}\right)-\sum_{j=1}^{N}\left(\bar{\omega}_{i j} R_{2}^{\beta_{i j}}+R_{2}^{\gamma_{i j}} \sup _{t} \int_{-\theta_{i j}}^{0} \kappa_{i j}(t, u) d u\right)\right. \\
& \left.-\frac{\bar{q}_{i}}{\underline{k}_{i}}\left(\bar{v}_{i} R_{2}^{\alpha_{i i}}+\bar{r}_{i}\right)-\bar{\wp}_{i i} R_{2}\right] \\
\geq & G(t)\left[\underline{\ell}_{i}-\sum_{j=1}^{N}\left(\bar{\varsigma}_{i j} R_{2}^{\alpha_{i j}}+\bar{v}_{i j} R_{2}^{\alpha_{i i}} R_{2}^{\alpha_{i j}}\right)-\sum_{j=1}^{N}\left(\bar{\omega}_{i j} R_{2}^{\beta_{i j}}+R_{2}^{\gamma_{i j}} \sup _{t} \int_{-\theta_{i j}}^{0} \kappa_{i j}(t, u) d u\right)-\frac{\bar{q}_{i}}{\underline{k}_{i}}\left(\bar{v}_{i} R_{2}^{\alpha_{i i}}+\bar{r}_{i}\right)\right]
\end{aligned}
$$

Integrating (6) over $J_{1}, t$ ) leads to

$$
G(t) \geq G\left(J_{1}\right) e^{L\left(t-J_{1}\right)}
$$

where

$$
L=\underline{\ell}_{i}-\sum_{j=1}^{N}\left(\bar{\wp}_{i j} R_{2}^{\alpha_{i j}}+\bar{v}_{i j} R_{2}^{\alpha_{i i}} R_{1}^{\alpha_{i j}}\right)-\sum_{j=1}^{N}\left(\bar{\omega}_{i j} R_{2}^{\beta_{i j}}+R_{2}^{\gamma_{i j}} \sup _{t} \int_{-\theta_{i j}}^{0} \kappa_{i j}(t, u) d u\right)-\frac{\bar{q}_{i}}{\underline{k}_{i}}\left(\bar{v}_{i} R_{2}^{\alpha_{i i}}+\bar{r}_{i}\right)
$$

By hypothesis (A5), for $t \rightarrow \infty$ we have $e^{L\left(t-J_{1}\right)} \rightarrow \infty$, which contradicts the fact that $G(t) \leq R_{1}$, for $t \geq J_{1}$.

$z_{i}^{\prime}(t) \geq z_{i}(t)\left[\underline{\ell}_{i}-\sum_{j=1, j \neq i}^{N}\left(\wp_{i j} R_{2}^{\alpha_{i j}}+\bar{v}_{i j} R_{2}^{\alpha_{i i}} R_{2}^{\alpha_{i j}}\right)-\sum_{j=1}^{N}\left(\bar{\omega}_{i j} R_{2}^{\beta_{i j}}+R_{2}^{\gamma_{i j}} \sup _{s} \int_{-\theta_{i j}}^{0} \kappa_{i j}(t, u) d u\right)-\frac{\bar{q}_{i}}{\underline{k}_{i}}\left(\bar{v}_{i} R_{2}^{\alpha_{i i}}+\bar{r}_{i}\right)-\wp_{i i}(t) z_{i}^{\alpha_{i i}}\right]$.

Let $y_{i}(t)=z_{i}^{\alpha_{i i}}(t)$, so

$y_{i}^{\prime}(t) \geq \alpha_{i i} y_{i}(t)\left[\underline{\ell}_{i}-\sum_{j=1, j \neq i}^{N}\left(\bar{\wp}_{i j} R_{2}^{\alpha_{i j}}+\bar{v}_{i j} R_{2}^{\alpha_{i i}} R_{2}^{\alpha_{i j}}\right)-\sum_{j=1}^{N}\left(\bar{\omega}_{i j} R_{2}^{\beta_{i j}}+R_{2}^{\gamma_{i j}} \sup _{s} \int_{-\theta_{i j}}^{0} \kappa_{i j}(t, u) d u\right)-\frac{\bar{q}_{i}}{k_{i}}\left(\bar{v}_{i} R_{2}^{\alpha_{i i}}+\bar{r}_{i}\right)-\wp_{i i}(t) y_{i}\right]$.

By the comparability theorem, we have

$$
\liminf _{t \rightarrow \infty} y_{i}(t) \geq\left(\max _{1 \leq i \leq N} \frac{\Theta}{\wp_{i i}}\right)^{\frac{1}{\alpha_{i i}}}=R_{1},
$$

where

$$
\Theta=\underline{\ell}_{i}-\sum_{j=1, j \neq i}^{N}\left(\bar{\wp}_{i j} R_{2}^{\alpha_{i j}}+\bar{v}_{i j} R_{2}^{\alpha_{i i}} R_{2}^{\alpha_{i j}}\right)-\sum_{j=1}^{N}\left(\bar{\omega}_{i j} R_{2}^{\beta_{i j}}+R_{2}^{\gamma_{i j}} \sup _{t} \int_{-\theta_{i j}}^{0} \kappa_{i j}(t, u) d u\right)-\frac{\bar{q}_{i}}{\underline{k}_{i}}\left(\bar{v}_{i} R_{2}^{\alpha_{i i}}+\bar{r}_{i}\right) .
$$




\section{Existence of the $(\mu, \eta)$-pseudo almost automorphic solutions}

Some results are established in this section as regards $(\mu, \eta)$-pseudo almost automorphic solutions existence remaining within $\Omega=\left\{Z \in P A A\left(\mathbb{R}, \mathbb{R}_{+}^{N}, \mu, \eta\right) \mid \frac{R_{1}}{R} \leq Z(t) \leq \frac{R_{2}}{R}\right\}$, where $0<R \leq R_{1}$.

Remark 5. One of the our contributions is the presentation of some new proofs that relate to the following Lemma.

Lemma 4. If $z(.) \in P A A\left(\mathbb{R}, \mathbb{R}_{+}, \mu, \eta\right)$ and $e(.) \in A A\left(\mathbb{R}, \mathbb{R}_{+}\right)$. Then $z(.-e().) \in P A A\left(\mathbb{R}, \mathbb{R}_{+}, \mu, \eta\right)$.

Proof. $z(.) \in P A A\left(\mathbb{R}, \mathbb{R}_{+}, \mu, \eta\right)$, using the composition theorem $z\left(\right.$.) is written as, $z=z_{1}+z_{2}$, where $z_{1}(.) \in$ $A A\left(\mathbb{R}, \mathbb{R}_{+}\right)$and $z_{2}(.) \in \varepsilon\left(\mathbb{R}, \mathbb{R}_{+}, \mu, \eta\right)$.

$$
z(t-e(t))=z_{1}(t-e(t))+z(t-e(t))-z_{1}(t-e(t)) .
$$

Next, it should be proven that $z_{1}(.-e().) \in A A\left(\mathbb{R}, \mathbb{R}_{+}\right)$. Let $H$ be a function defined by $H(t, r)=z_{1}(t-r)$. It should be also proven that $H \in A A_{U}\left(\mathbb{R} \times \mathbb{R}, \mathbb{R}_{+}\right)$. Firstly, since $z_{1}(.) \in A A\left(\mathbb{R}, \mathbb{R}_{+}\right)$and space $A A\left(\mathbb{R}, \mathbb{R}_{+}\right)$is invariant through the use of translation then $z_{1}(.-r) \in A A\left(\mathbb{R}, \mathbb{R}_{+}\right)$. In addition, $z_{1}(t-$.$) is then z_{1}(t-$.$) is$ continuous and by Heine's theorem, $z_{1}(t-$.) is uniformly continuous in every compact $\mathcal{K}$ of $\mathbb{R}$.

Hence, by Theorem (1), $H \in A A_{U}\left(\mathbb{R} \times \mathbb{R}, \mathbb{R}_{+}\right)$. Define the Nemytskii's operator $N_{H}$ through the utilization of

$$
\begin{aligned}
N_{H}: A A(\mathbb{R}, \mathbb{R}) & \rightarrow A A(\mathbb{R}, \mathbb{R}) \\
e & \mapsto N_{H}(e):=[t \mapsto f(e(t), t)] .
\end{aligned}
$$

Since $H \in A A_{U}\left(\mathbb{R} \times \mathbb{R}, \mathbb{R}_{+}\right)$, then $N_{H}$ can be well defined and continuous. By the same token, $e(.) \in A A\left(\mathbb{R}, \mathbb{R}_{+}\right)$, then by Theorem (2), the function $t \mapsto H(t, e(t))=z_{1}(t-e(t))$ is almost automorphic.

Now, we show that $z(t-e(t))-z_{1}(t-e(t)) \in \varepsilon\left(\mathbb{R}, \mathbb{R}_{+}, \mu, \eta\right)$.

$$
\begin{aligned}
& \lim _{w \rightarrow \infty} \frac{1}{\eta([-w, w])} \int_{-w}^{w}\left|z(t-e(t))-z_{1}(t-e(t))\right| d \mu(t) \\
= & \lim _{w \rightarrow \infty} \frac{1}{\eta([-w, w])} \int_{-w}^{w}\left|z_{2}(t-e(t))\right| d \mu(t) \\
\leq & \lim _{w \rightarrow \infty} \frac{1}{\eta([-w, w])} \int_{-(w+\bar{e})}^{w+\bar{e}}\left|z_{2}(t)\right| d \mu(t+e(t)) \\
\leq & \lim _{w \rightarrow \infty} \frac{\eta([-w-\bar{e}, w+\bar{e}])}{\eta([-w, w])} \frac{1}{\eta([-w-\bar{e}, w+\bar{e}])} \int_{-(w+\bar{e})}^{w+\bar{e}}\left|z_{2}(t)\right| \lambda(t) d \mu(t) \\
\leq & \lim _{w \rightarrow \infty} \frac{\eta([-w-\bar{e}, w+\bar{e}])}{\eta([-w, w])} \sup _{\xi \in[-(w+\bar{e}), w+\bar{e}]}^{\lambda(\xi)} \frac{1}{\eta([-w-\bar{e}, w+\bar{e}])} \int_{-(w+\bar{e})}^{w+\bar{e}}\left|z_{2}(t)\right| d \mu(t) .
\end{aligned}
$$

Since $\eta$ satisfies (A1) and $z_{2}(.) \in \varepsilon\left(\mathbb{R}, \mathbb{R}_{+}, \mu, \eta\right)$. Thus $z(t-e(t))-z_{1}(t-e(t)) \in \varepsilon\left(\mathbb{R}, \mathbb{R}_{+}, \mu, \eta\right)$. So $z(.-e().) \in$ $\operatorname{PAA}\left(\mathbb{R}, \mathbb{R}_{+}, \mu, \eta\right)$.

Lemma 5. Under (A1)-(A4), for every $1 \leq j \leq N$ the function $\mathfrak{G}_{j}: t \rightarrow \int_{-\theta_{i j}}^{0} \kappa_{i j}(t, s)\left(z_{j}\right)^{\gamma_{i j}}(t+u) d u$ is affiliated with $P A A\left(\mathbb{R}, \mathbb{R}_{+}, \mu, \eta\right)$.

Proof. The function $t \rightarrow\left(z_{j}\right)^{\gamma_{i j}}(t+s)$ is $(\mu, \eta)$-pseudo almost automorphic function since $\operatorname{PAA}\left(\mathbb{R}_{1}, \mathbb{R}_{+}, \mu, \eta\right)$ is invariant by translation. Using composition Theorem $(4), \kappa_{i j}(., s),\left(z_{j}\right)^{\gamma_{i i}}($.$) can be expressed as follows$

$$
\kappa_{i j}(t, s)=\kappa_{i j}^{1}(t, s)+\kappa_{i j}^{2}(t, s), z_{j}^{\gamma_{i j}}(t+s)=z_{1 j}(t+s)+z_{2 j}(t+s)
$$


where $\kappa_{i j}^{1}(., s), z_{1 j}(.+s) \in A A\left(\mathbb{R}, \mathbb{R}_{+}\right)$and $\kappa_{i j}^{2}(., s), z_{2 j}(.+s) \in \varepsilon\left(\mathbb{R}, \mathbb{R}_{+}, \mu, \eta\right)$. Then,

$$
\begin{aligned}
\mathfrak{G}_{j}(t) & =\int_{-\theta_{i j}}^{0}\left[\kappa_{i j}^{1}(t, \iota)+\kappa_{i j}^{2}(t, \iota)\right]\left[z_{1 j}(t+\iota)+z_{2 j}(t+\iota)\right] d \iota \\
& =\int_{-\theta_{i j}}^{0} \kappa_{i j}^{1}(t, \iota) z_{1 j}(t+\iota) d \iota+\int_{-\theta_{i j}}^{0}\left[\kappa_{i j}^{1}(t, \iota) z_{2 j}(t+\iota)+\kappa_{i j}^{2}(t, \iota) z_{1 j}(t+\iota)+\kappa_{i j}^{2}(t, \iota) z_{2 j}(t+\iota)\right] d \iota \\
& =\mathfrak{G}_{i}^{1}(t)+\mathfrak{G}_{i}^{2}(t),
\end{aligned}
$$

where

$$
\begin{aligned}
& \mathfrak{G}_{i}^{1}(t)=\int_{-\theta_{i j}}^{0} \kappa_{i j}^{1}(t, \iota) z_{1 j}(t+\iota) d \iota, \\
& \mathfrak{G}_{i}^{2}(t)=\int_{-\theta_{i j}}^{0}\left[\kappa_{i j}^{1}(t, \iota) z_{2 j}(t+\iota)+\kappa_{i j}^{2}(t, s) z_{1 j}(t+\iota)+\kappa_{i j}^{2}(t, \iota) z_{2 j}(t+\iota)\right] d \iota .
\end{aligned}
$$

Firstly, it is clear that $\mathfrak{G}_{j}^{1}(),. \mathfrak{G}_{j}^{2}($.$) are continuous. Afterwards, it must be proven that \mathfrak{G}_{j}^{1}(.) \in A A\left(\mathbb{R}, \mathbb{R}_{+}\right)$. Since $\kappa_{i j}^{1}(., s), z_{1 j}($.$) are almost automorphic, it is possible to extract for each sequence of real numbers \left(j_{n}\right)$ a subsequence $\left(l_{n}\right)$ in a certain manner that for $n \geq N$, we have

$$
\lim _{n \rightarrow \infty} \kappa_{i j}^{1}\left(t+l_{n}, \iota\right)=v_{i j}(t, \iota), \lim _{n \rightarrow \infty} v_{i j}\left(t-l_{n}, \iota\right)=\kappa_{i j}^{1}(t, \iota)
$$

for all $\iota \in\left[-\theta_{i j}, 0\right]$, and

$$
\lim _{n \rightarrow \infty} z_{1 j}\left(t+l_{n}+\iota\right)=z_{j}^{1}(t+\imath), \lim _{n \rightarrow \infty} z_{j}^{1}\left(t+l_{n}+\iota\right)=z_{1 j}(t+\iota)
$$

uniformly for $t \in \mathbb{R}$. From (7) and (8), there exist a positive integer $N$, in a certain way that

$$
\left|\kappa_{i j}^{1}\left(t+l_{n}, \iota\right)-v_{i j}(t, \iota)\right|<\frac{\varepsilon}{2 e\left\|z_{j}\right\|_{\infty}},\left|z_{1 j}\left(t+l_{n}+\iota\right)-z_{j}^{1}(t+\iota)\right|<\frac{\varepsilon}{2 e\left\|v_{i j}\right\|_{\infty}} .
$$

Pose $\tilde{J}_{j}(t)=\int_{-\theta_{i j}}^{0} v_{i j}(t, \iota)\left(z_{j}^{1}\right)^{\gamma_{i j}}(t+\imath) d \iota$. On the other hand,

$$
\begin{aligned}
\left|\mathfrak{G}_{j}^{1}\left(t+l_{n}\right)-\tilde{J}_{j}(t)\right| & =\left|\int_{-\theta_{i j}}^{0}\left[\kappa_{i j}^{1}\left(t+L_{n}, \iota\right)-v_{i j}(t, \iota)\right] z_{1 j}\left(t+l_{n}+\iota\right) d \iota+\int_{-\theta_{i j}}^{0} v_{i j}(t, \iota)\left[\left(z_{1 j}\right)\left(t+l_{n}+\iota\right)-\left(z_{j}^{1}\right)(t+\iota)\right] d \iota\right| \\
& \leq \int_{-\theta_{i j}}^{0}\left|\kappa_{i j}^{1}\left(t+l_{n}, \iota\right)-v_{i j}(t, \iota)\left\|z_{1 j}\left(t+l_{n}+\iota\right)\left|d \iota+\int_{-\theta_{i j}}^{0}\right| v_{i j}(t, \iota)\right\|\left(z_{1 j}\right)\left(t+l_{n}+\iota\right)-\left(\begin{array}{l}
1 \\
j
\end{array}\right)(t+\iota)\right| d \iota \\
& <\frac{\varepsilon}{2\left\|e_{1 j}\right\|_{\infty}}\left\|z_{1 j}\right\|_{\infty}+\left\|v_{i j}\right\|_{\infty} \int_{-\theta_{i j}}^{0} \frac{\varepsilon}{2 e\left\|v_{i j}\right\|_{\infty}} d \iota \\
& <\varepsilon .
\end{aligned}
$$

It is proven in the same approach that, for all $t \in \mathbb{R}$

$$
\lim _{n \rightarrow \infty} \tilde{J}_{j}\left(t-l_{n}\right)=\mathfrak{G}_{j}^{1}(t),
$$

which proves that $\mathfrak{G}_{j}^{1}(.) \in A A\left(\mathbb{R}, \mathbb{R}_{+}\right)$. 
After that, we can show that $\mathfrak{G}_{j}^{2}(.) \in \varepsilon\left(\mathbb{R}, \mathbb{R}_{+}, \mu, \eta\right)$, for every $j=1, \ldots, N$. That is to say, we have to prove that $\lim _{w \rightarrow \infty} \frac{1}{\eta([-w, w])} \int_{-w}^{w}\left|\mathfrak{G}_{j}^{2}(t)\right| d \mu(t)=0$.

$$
\begin{aligned}
\lim _{w \rightarrow \infty} \frac{1}{\eta([-w, w])} \int_{-w}^{w}\left|G_{j}^{2}(t)\right| d \mu(t) \leq & \lim _{w \rightarrow \infty} \frac{1}{\eta([w, w])} \int_{-w}^{w} \int_{-\theta_{i j}}^{0}\left|\kappa_{i j}^{1}(t, \iota) \| z_{2 j}(t+\iota)\right| \\
& +\left|\kappa_{i j}^{2}(t, \iota)\right|\left|z_{1 j}(t+\iota)\right|+\left|\kappa_{i j}^{2}(t, \iota)\right|\left|z_{2 j}(t+\iota)\right| d \mu(t) d \iota \\
\leq & \lim _{w \rightarrow \infty} \frac{1}{\eta([-w, w])}\left[\int _ { - w } ^ { w } \left(\left\|\kappa_{i j}^{1}\right\|_{\infty} \int_{-\theta_{i j}}^{0}\left|z_{2 j}(t+\iota)\right| d \iota\right.\right. \\
& \left.\left.+\left\|z_{1 j}\right\|_{\infty} \int_{-\theta_{i j}}^{0}\left|\kappa_{i j}^{2}(t, \iota)\right| d \iota+\left\|z_{2 j}\right\|_{\infty} \int_{-\theta_{i j}}^{0}\left|\kappa_{i j}^{2}(t, \iota)\right| d \iota\right) d \mu(t)\right] \\
\leq & \lim _{w \rightarrow \infty} \frac{1}{\eta([-w, w])}\left[\int_{-\theta_{i j}}^{0}\left\|z_{1 j}\right\|_{\infty} \int_{-w}^{w}\left|\kappa_{i j}^{2}(t, \iota)\right| d \mu(t) d \iota\right] \\
& +\lim _{w \rightarrow \infty} \frac{1}{\eta([-w, w])}\left[\int_{-\theta_{i j}}^{0}\left\|z_{2 j}\right\|_{\infty} \int_{-w}^{w}\left|\kappa_{i j}^{2}(t, \iota)\right| d \mu(t) d \iota\right] \\
& +\left\|\kappa_{i j}^{1}\right\|_{\infty} \lim _{w \rightarrow \infty} \frac{1}{\eta([-w, w])} \int_{-\theta_{i j}}^{0} \int_{-w}^{w}\left|z_{2 j}(t+\iota)\right| d \mu(t) d \iota .
\end{aligned}
$$

Since $\mu$ satisfies (A1)-(ii) and $\kappa_{i j}^{2}(.,),. z_{2 j}(.) \in \varepsilon\left(\mathbb{R}, \mathbb{R}_{+}, \mu, \eta\right)$, and by the Lebesgue dominated convergence theorem, we are able to get

$$
\lim _{w \rightarrow \infty} \frac{1}{v([-w, w])} \int_{-w}^{w}\left|\mathfrak{G}_{j}^{2}(t)\right| d \mu(t)=0 .
$$

Consequently, $\mathfrak{G}_{j}($.$) is (\mu, \eta)$-pseudo almost automorphic function.

Lemma 6. Under $(A 1)-(A 3)$. For every $1 \leq i \leq N$, function $t \mapsto\left(\Psi z_{i}\right)(t)$ is affiliated with $P A A(\mathbb{R}, \mathbb{R}, \mu, \eta)$.

Proof. Indeed, for every $1 \leq i \leq N$, function $\Lambda_{i}: t \mapsto r_{i}(t)+v_{i}(t) z_{i}^{\alpha_{i i}}(t)$ is $(\mu, \eta)$-pseudo almost automorphic. Using composition Theorem (4), $\Lambda_{i}($.$) is expressed as$

$$
\Lambda_{i}(t)=\Lambda_{1 i}(t)+\Lambda_{2 i}(t)
$$

with $\Lambda_{1 i}(.) \in A A(\mathbb{R}, \mathbb{R}), \Lambda_{2 i}(.) \in \varepsilon\left(\mathbb{R}, \mathbb{R}^{N}, \mu, \eta\right)$. Therefore

$$
\left(\Psi x_{i}\right)(t)=A_{i}(t)+B_{i}(t)
$$

with

$$
A_{i}(t)=\int_{\infty}^{t} e^{-\int_{s}^{t} k_{i}(u) d u} \Lambda_{1 i}(s) d s, B_{i}(t)=\int_{\infty}^{t} e^{-\int_{s}^{t} k_{i}(u) d u} \Lambda_{2 i}(s) d s .
$$

Since $\Lambda_{1 i}(),. k_{i}($.$) are almost automorphic, we will be able to extract for each sequence of real numbers \left(p_{n}\right)$, a subsequence $\left(l_{n}\right)$ in a certain degree that

$$
\begin{aligned}
& \lim _{n \rightarrow \infty} \Lambda_{1 i}\left(t+l_{n}\right)=K_{1 i}(t), \lim _{n \rightarrow \infty} k_{i}\left(t+l_{n}\right)=J_{i}(t) \\
& \lim _{n \rightarrow \infty} K_{1 i}\left(t-l_{n}\right)=\Lambda_{1 i}(t), \lim _{n \rightarrow \infty} J_{i}\left(t-l_{n}\right)=k_{i}(t)
\end{aligned}
$$


or $t \in \mathbb{R}$. Note that $W_{i}(t)=\int_{\infty}^{t} e^{-\int_{t}^{s} J_{i}(u) d u} K_{1 i}(s) d s$. For $t \geq 0$, we get

$$
\begin{aligned}
\left|A_{i}\left(t+l_{n}\right)-W_{i}(t)\right|= & \left|\int_{\infty}^{t+l_{n}} e^{-\int_{t+l_{n}}^{s} k_{i}(u) d u} \Lambda_{1 i}(s) d s-\int_{\infty}^{t} e^{-\int_{s}^{t} J_{i}(u) d u} K_{1 i}(s) d s\right| \\
\leq & \int_{\infty}^{t}\left|e^{-\int_{s}^{t} k_{i}\left(u+l_{n}\right) d u} \Lambda_{1 i}\left(s+l_{n}\right)-e^{-\int_{t}^{u} J_{i}(u) d u} K_{1 i}(s)\right| d s \\
\leq & \int_{\infty}^{t} e^{-\int_{s}^{t} k_{i}\left(u+l_{n}\right) d u}\left|\Lambda_{1 i}\left(s+l_{n}\right)-K_{1 i}(s)\right| d s \\
& +\int_{\infty}^{t}\left\|K_{1 i}\right\|_{\infty}\left|e^{-\int_{s}^{t} k_{i}\left(u+l_{n}\right) d u}-e^{-\int_{s}^{t} J_{i}(u) d u}\right| d s .
\end{aligned}
$$

There exists $\theta \in] 0,1[$ in a way that

$$
\begin{aligned}
\left|A_{i}\left(t+l_{n}\right)-W_{i}(t)\right| \leq & \int_{\infty}^{t} e^{-(s-t) \underline{k}_{i} d u}\left|\Lambda_{1 i}\left(s+l_{n}\right)-K_{1 i}(s)\right| d s+\int_{\infty}^{t}\left\|K_{1 i}\right\|_{\infty} \\
& \left|\int_{s}^{t}\right| k_{i}\left(u+l_{n}\right)-J_{i}(u) d u \mid e^{-\int_{s}^{t} k_{i}\left(u+l_{n}\right) d u-\theta\left(\int_{t}^{s} e_{i}\left(u+s_{n}\right)-J_{i}(u) d u\right)} d s \\
\leq & \int_{\infty}^{t} e^{-(t-s) \underline{e}_{i} d u}\left|\Lambda_{1 i}\left(s+s_{n}\right)-K_{1 i}(s)\right| d s \\
& +\int_{\infty}^{t}\left\|K_{1 i}\right\|_{\infty} \| \int_{s}^{t}\left|e_{i}\left(u+s_{n}\right)-J_{i}(u) d u\right| e^{-(t-s) \underline{e}_{i}} d s \\
& =\int_{\infty}^{t} Q_{i}(t, s) d s+\int_{\infty}^{t} E_{i}(t, s) d s,
\end{aligned}
$$

where

$$
\begin{aligned}
Q_{i}(t, s) & =e^{-(t-s) \underline{k}_{i} d u}\left|\Lambda_{1 i}\left(s+l_{n}\right)-K_{1 i}(s)\right|, \\
E_{i}(t, s) & =\left\|K_{1 i}\right\|_{\infty} \| \int_{s}^{t} k_{i}\left(u+l_{n}\right)-J_{i}(u) d u \mid e^{-(t-s) \underline{k_{i}} d u} .
\end{aligned}
$$

Using Lebesgue dominated convergence theorem, we can get

$$
\lim _{t \rightarrow \infty} A_{i}\left(t+l_{n}\right)=W_{i}(t), \text { for all } t \in \mathbb{R} .
$$

In a similar way

$$
\lim _{t \rightarrow \infty} W_{i}\left(t-l_{n}\right)=A_{i}(t), \text { for all } t \in \mathbb{R} .
$$

Consequently, $A_{i}(.) \in A A\left(\mathbb{R}, \mathbb{R}_{+}\right)$. Next, we shall prove $B_{i}(.) \in \varepsilon\left(\mathbb{R}, \mathbb{R}_{+}, \mu, \eta\right)$.

$$
\lim _{w \rightarrow \infty} \frac{1}{\eta([-w, w])} \int_{-w}^{w}\left|B_{i}(t)\right| d \mu(t)=\lim _{w \rightarrow \infty} \frac{1}{\eta([-w, w])} \int_{-w}^{w}\left|\int_{\infty}^{t} e^{\left(-\int_{l}^{t} k_{i}(u) d u\right)} \Lambda_{2 i}(\iota) d \iota\right| d \mu(t)
$$




$$
\begin{aligned}
& \leq \lim _{w \rightarrow \infty} \frac{1}{\eta([-w, w])} \int_{-w}^{w} \int_{\infty}^{t}\left|\Lambda_{2 i}(\iota)\right| e^{-(t-\iota) \underline{k}_{i}} d \iota d \mu(t) \\
& \leq \lim _{w \rightarrow \infty} \frac{1}{\eta([-w, w])} \int_{-w}^{w} \int_{0}^{\infty} e^{-u \underline{k}_{i}}\left|\Lambda_{2 i}(t-\iota)\right| d \iota d \mu(t) \\
& \leq \int_{0}^{\infty} e^{-u \underline{k}_{i}}\left(\lim _{T \rightarrow \infty} \frac{1}{\eta([-w, w])} \int_{-w}^{w}\left|\Lambda_{2 i}(t-\iota)\right| d \mu(t)\right) d \iota .
\end{aligned}
$$

Since $v_{i} \in \varepsilon\left(\mathbb{R}, \mathbb{R}_{+} \mu, \eta\right)$ and by hypothesis (A1), the function $B_{i}(.) \in \varepsilon\left(\mathbb{R}, \mathbb{R}_{+}, \mu, \eta\right)$.

Theorem 6. Assume that $(A 1)-(A 5)$ hold. The nonlinear operator $\Xi$ can be defined follows, for each $Z=$ $\left(z_{1}, \ldots, z_{N}\right) \in P A A\left(\mathbb{R}, \mathbb{R}^{N}, \mu, \eta\right), \Xi Z(t):=\left(\left(\Xi z_{1}\right)(t), \ldots,\left(\Xi z_{N}\right)(t)\right)$ where

$$
\left(\Xi z_{i}\right)(t)=\int_{t}^{\infty} e^{-\int_{t}^{s} \ell_{i}(u) d u} \digamma_{i}(s) d s,
$$

and

$$
\begin{aligned}
\digamma_{i}(s)= & z_{i}(s)\left[\sum_{j=1}^{N} \wp_{i j}(s) z_{j}^{\alpha_{i j}}(s)+\sum_{j=1}^{N} \omega_{i j}(s) z_{j}^{\beta_{i j}}\left(s-e_{i j}(s)\right)+q_{i}(s)\left(\Psi z_{i}\right)(s)\right. \\
& \left.+\sum_{j=1, j \neq i}^{N} v_{i j}(s) z_{i}^{\alpha_{i i}}(s) z_{j}^{\alpha_{i j}}(s)+\sum_{j=1}^{N} \int_{-\theta_{i j}}^{0} \kappa_{i j}(s, u) z_{j}^{\gamma_{i j}}(u+s) d u\right]
\end{aligned}
$$

for all $1 \leq i \leq N$. Then $\Xi$ maps to $P A A\left(\mathbb{R}, \mathbb{R}_{+}, \mu, \eta\right)$.

Proof. For every $1 \leq i \leq N$, function $\digamma_{i}: s \mapsto \digamma_{i}(s)$ is $(\mu, \eta)$-pseudo almost automorphic. Then, for every $1 \leq i \leq N, \digamma_{i}$ can be expressed as

$$
\digamma_{i}(s)=\digamma_{i}^{1}(s)+\digamma_{i}^{2}(s)
$$

with $\digamma_{i}^{1}(.) \in A A\left(\mathbb{R}, \mathbb{R}_{+}\right)$and $\digamma_{i}^{2} \in \varepsilon\left(\mathbb{R}, \mathbb{R}_{+}, \mu, \eta\right)$. As a result,

$$
\left(\Xi z_{i}\right)(t)=\left(\Xi \digamma_{i}^{1}\right)(t)+\left(\Xi \digamma_{i}^{2}\right)(t) .
$$

Let consider $\left(p_{n}\right)$ a sequence of real numbers. Since $\digamma_{i}^{1}, \ell_{i}$ are almost automorphic functions, we are capable of extracting a subsequence $\left(a_{n}\right)$ of $\left(p_{n}\right)$ in a certain degree that

$$
\begin{aligned}
\lim _{n \rightarrow \infty} \ell_{i}\left(t+a_{n}\right) & =r_{i}^{1}(t), \lim _{n \rightarrow \infty} r_{i}^{1}\left(t-a_{n}\right)=r_{i}(t) \text { for all } t \in \mathbb{R} \\
\lim _{n \rightarrow \infty} \digamma_{i}^{1}\left(t+a_{n}\right) & =X_{i}(t), \lim _{n \rightarrow \infty} X_{i}\left(t-a_{n}\right)=\digamma_{i}^{1}(t) \text { for all } t \in \mathbb{R} .
\end{aligned}
$$

Pose

$$
\left(\Xi X_{i}\right)(t)=\int_{t}^{\infty} e^{-\int_{t}^{s} r_{i}^{1}(u) d u} X_{i}(s) d s
$$


Afterwards,

$$
\begin{aligned}
\left(\Xi \digamma_{i}^{1}\right)\left(t+a_{n}\right)-\left(\Xi X_{i}\right)(t)= & \int_{t+a_{n}}^{\infty} e^{-\int_{t+a_{n}}^{t} \ell_{i}(u) d u} \digamma_{i}(\iota) d \iota-\int_{t}^{\infty} e^{-\int_{t}^{t} r_{i}^{1}(u) d u} X_{i}(\iota) d \iota \\
= & \int_{t}^{\infty} e^{-\int_{t}^{\iota} \ell_{i}\left(u+a_{n}\right) d u} \digamma_{i}\left(\iota+a_{n}\right) d s-\int_{t}^{\infty} e^{-\int_{t}^{t} r_{i}^{1}(u) d u} X_{i}(\iota) d \iota \\
= & \int_{t}^{\infty} e^{-\int_{t}^{t} \ell_{i}\left(u+a_{n}\right) d u}\left[\digamma_{i}\left(\iota+a_{n}\right)-X_{i}(\iota)\right] d \iota \\
& +\int_{t}^{\infty}\left[e^{-\int_{t}^{t} \ell_{i}\left(u+a_{n}\right) d u}-e^{-\int_{t}^{t} r_{i}^{1}(u) d u}\right] X_{i}(\iota) d \iota .
\end{aligned}
$$

Thus,

$$
\begin{aligned}
\left|\left(\Xi \digamma_{i}^{1}\right)\left(t+a_{n}\right)-\left(\Xi X_{i}\right)(t)\right| \leq & \int_{t}^{\infty} e^{-(\iota-t) \underline{\ell}_{i}}\left|\digamma_{i}\left(\iota+a_{n}\right)-X_{i}(\iota)\right| d \iota \\
& +\left\|X_{i}\right\|_{\infty} \int_{t}^{\infty}\left|e^{-\int_{t}^{l} \ell_{i}\left(u+a_{n}\right) d u}-e^{-\int_{t}^{l} r_{i}^{1}(u) d u}\right| d \iota .
\end{aligned}
$$

Hence, we have $\theta \in] 0,1[$ in a certain way

$$
\begin{aligned}
& \left|\left(\Xi \digamma_{i}^{1}\right)\left(t+a_{n}\right)-\left(\Xi X_{i}\right)(t)\right| \leq \int_{t}^{\infty} e^{-(t-l) \underline{\ell}_{i}}\left|\digamma_{i}\left(l+a_{n}\right)-X_{i}(l)\right| d \iota \\
& +\left\|X_{i}\right\|_{\infty} \int_{t}^{\infty}\left|\int_{t}^{t}\left[\ell_{i}\left(u+a_{n}\right)-r_{i}^{1}(u)\right] d u\right| \\
& e^{-\int_{t}^{t} \ell_{i}\left(u+a_{n}\right) d u+\theta\left(\int_{t}^{t} r_{i}^{1}(u) d u-\int_{t}^{\iota} \ell_{i}\left(u+s_{n}\right) d u\right)} d \iota \\
& \leq \int_{t}^{\infty} e^{-(\iota-t) \underline{\ell}_{i}}\left|\digamma_{i}\left(\iota+a_{n}\right)-X_{i}(\iota)\right| d \iota \\
& +\left\|X_{i}\right\|_{\infty} \int_{t}^{\infty}\left|\int_{t}^{t}\left[\ell_{i}\left(u+a_{n}\right)-r_{i}^{1}(u)\right] d u\right| e^{-(t-t) \underline{\ell}_{i}} \\
& e^{-\theta\left(\int_{t}^{t} r_{i}^{1}(u) d u-\int_{t}^{t} \ell_{i}\left(u+a_{n}\right) d u\right)} d \iota \\
& \leq \int_{t}^{\infty} e^{-(\iota-t) \underline{\ell}_{i}}\left|\digamma_{i}\left(\iota+a_{n}\right)-X_{i}(\iota)\right| d \iota \\
& +\left\|X_{i}\right\|_{\infty} \int_{t}^{\infty} \int_{t}^{\iota}\left|\ell_{i}\left(u+a_{n}\right)-r_{i}^{1}(u)\right| d u e^{-(\iota-t) \underline{\ell}_{i}} d \iota \\
& =\int_{t}^{\infty} K_{i}(t, \iota) d \iota+\int_{t}^{\infty} G_{i}(t, \iota) d \iota,
\end{aligned}
$$

with

$$
\begin{aligned}
& K_{i}(t, \iota)=e^{-(\iota-t) \ell_{i}}\left|\digamma_{i}\left(\iota+a_{n}\right)-X_{i}(\iota)\right|, \\
& G_{i}(t, \iota)=e^{-(\iota-t) \underline{\ell}_{i}} \int_{t}^{\iota}\left|\ell_{i}\left(u+a_{n}\right)-r_{i}^{1}(u)\right| d u .
\end{aligned}
$$


Using Lebesgue dominated convergence theorem, we are able to obtain

$$
\lim _{n \rightarrow \infty}\left(\Xi \digamma_{i}^{1}\right)\left(t+a_{n}\right)=\left(\Xi X_{i}\right)(t) .
$$

The same approach proves that for every $t \in \mathbb{R}$

$$
\lim _{n \rightarrow \infty}\left(\Xi X_{i}\right)\left(t-a_{n}\right)=\left(\Xi \digamma_{i}^{1}\right)(t),
$$

which implies that $\left(\Xi \digamma_{i}^{1}\right)(.) \in A A\left(\mathbb{R}, \mathbb{R}_{+}\right)$. Let us prove $\left(\Xi \digamma_{i}^{2}\right)(.) \in \varepsilon\left(\mathbb{R}, \mathbb{R}_{+}, \mu, \eta\right)$

$$
\begin{aligned}
& \lim _{w \rightarrow \infty} \frac{1}{\eta([-w, w])} \int_{-w}^{w}\left|\left(\Xi \digamma_{i}^{2}\right)(t)\right| d \mu(t) \leq \lim _{w \rightarrow \infty} \frac{1}{\eta([-w, w])} \int_{-w}^{w} \int_{t}^{\infty}\left|\digamma_{i}^{2}(\iota)\right| e^{\left(-\int_{t}^{t} \ell_{i}(u) d u\right)} d \iota d \mu(t) \\
& \leq \lim _{w \rightarrow \infty} \frac{1}{\eta([-w, w])} \int_{-w}^{w} \int_{t}^{w} e^{-(\iota-t) \underline{\ell}_{i}}\left|\digamma_{i}^{2}(\iota)\right| d \iota d \mu(t) \\
& +\lim _{w \rightarrow \infty} \frac{1}{\eta([-w, w])} \int_{-w}^{w} \int_{w}^{\infty} e^{-(\iota-t) \underline{\ell}_{i}}\left|\digamma_{i}^{2}(\iota)\right| d \iota d \mu(t) \\
& \leq \chi_{1}+\chi_{2} \text {, }
\end{aligned}
$$

where

$$
\begin{aligned}
& \chi_{1}=\lim _{w \rightarrow \infty} \frac{1}{\eta([-w, w])} \int_{-w}^{w} \int_{t}^{w} e^{-(\iota-t) \underline{\ell}_{i}}\left|\digamma_{i}^{2}(\iota)\right| d \iota d \mu(t), \\
& \chi_{2}=\lim _{w \rightarrow \infty} \frac{1}{\eta([-w, w])} \int_{-w}^{w} \int_{w}^{\infty} e^{-(\iota-t) \underline{\ell}_{i}}\left|\digamma_{i}^{2}(\iota)\right| d \iota d \mu(t) .
\end{aligned}
$$

It remains to show $\chi_{1}=\chi_{2}=0$.

$$
\begin{aligned}
\chi_{1} & \leq \lim _{w \rightarrow \infty} \frac{1}{\eta([-w, w])} \int_{-w}^{w} \int_{0}^{w-t} e^{-u \underline{\ell}_{i}}\left|\digamma_{i}^{2}(u+t)\right| d u d \mu(t) \\
& \leq \lim _{w \rightarrow \infty} \frac{1}{\eta([-w, w])} \int_{0}^{\infty} e^{-u \underline{\ell}_{i}} \int_{-(w+u)}^{w-u}\left|\digamma_{i}^{2}(t)\right| d \mu u(t) d u \\
& \leq \int_{0}^{\infty} e^{-u \underline{\ell}_{i}}\left(\lim _{w \rightarrow \infty}\left(\frac{\beta}{\eta([-w, w])} \int_{-w-u}^{w+u}\left|\digamma_{i}^{2}(\iota)\right| d \mu(\iota)\right) d u .\right.
\end{aligned}
$$

Actually, $\digamma_{i}^{2}(.) \in \varepsilon\left(\mathbb{R}, \mathbb{R}_{+} \mu, \eta\right)$, so $\chi_{1}=0$. On the other hand,

$$
\begin{aligned}
\chi_{2} \leq & \lim _{w \rightarrow \infty} \frac{1}{\eta([-w, w])} \int_{-w}^{w} \int_{w}^{\infty} e^{-(\iota-t) \underline{\ell}_{i}}\left|\digamma_{i}^{2}(\iota)\right| d \iota d \mu(t) \\
\leq & \left|\digamma_{i}^{2}\right|_{\infty} \lim _{w \rightarrow \infty} \frac{1}{\eta([-w, w])} \int_{-w}^{w} \int_{w}^{\infty} e^{-(\iota-t) \underline{\ell}_{i}} d \iota d \mu(t) \\
\leq & \left|\digamma_{i}^{2}\right|_{\infty} \lim _{w \rightarrow \infty} \frac{1}{\eta([-w, w])} \int_{-w}^{w} e^{t \underline{\ell}_{i}}\left(\int_{w}^{\infty} e^{-l \underline{\ell}_{i}} d \iota\right) d \mu(t) \\
& =\frac{\left|\digamma_{i}^{2}\right|_{\infty}}{\underline{\ell}_{i}} \lim _{w \rightarrow \infty} \frac{e^{-w \underline{\ell}_{i}}}{\eta([-w, w])} \int_{-w}^{w} e^{t \underline{\ell}_{i}} d \mu(t) \\
\left.\underline{\ell}_{i}\right|_{\infty} & \lim _{w \rightarrow \infty} e^{-2 w \underline{\ell}_{i}} \frac{\mu([-w, w])}{\eta([-w, w])} .
\end{aligned}
$$


By hypothesis (A1)-(i), we obtain

$$
\chi_{2} \leq \frac{\left|\digamma_{i}^{2}\right|_{\infty}}{\underline{\ell}_{i}} \sup _{\xi} \frac{\mu([-\xi, \xi])}{\eta([-\xi, \xi])} \lim _{w \rightarrow \infty} e^{-2 w \underline{\ell}_{i}}=0 .
$$

Hence showing beyond doubt that the operator $\Xi$ maps into itself.

Theorem 7. Under the hypothesis (A1) - (A5), equation (2) at least has in $\Omega$ a ( $\mu, \eta)$-pseudo almost automorphic solutions.

Proof. $\Omega$ is clearly one closed convex subset. Now it should be proven that $\Xi$ is a mapping from $\Omega$ to $\Omega$. In fact, using theorem (6) $\Xi \in P A A\left(\mathbb{R}, \mathbb{R}^{N}, \mu, \eta\right)$. Indeed, for $Z \in P A A\left(\mathbb{R}, \mathbb{R}_{+}^{N}, \mu, \eta\right)$,

$$
\left(\Xi z_{i}\right)(t) \geq \int_{t}^{\infty} e^{-(\iota-t) \bar{\ell}_{i}}\left|\digamma_{i}(\iota)\right| d \iota,
$$

where

$$
\begin{aligned}
\digamma_{i}(\iota)= & z_{i}(\iota)\left[\sum_{j=1}^{N} \wp_{i j}(\iota) z_{j}^{\alpha_{i j}}(\iota)+\sum_{j=1}^{N} \omega_{i j}(\iota) z_{j}^{\beta_{i j}}\left(\iota-z_{i j}(\iota)\right)+q_{i}(\iota)\left(\Psi z_{i}\right)(\iota)\right. \\
& \left.+\sum_{j=1, j \neq i}^{N} v_{i j}(s) z_{i}^{\alpha_{i i}}(\iota) z_{j}^{\alpha_{i j}}(\iota)+\sum_{j=1}^{N} \int_{-\theta_{i j}}^{0} \kappa_{i j}(\iota, u) z_{j}^{\gamma_{i j}}(u+\iota) d u\right],
\end{aligned}
$$

then

$$
\left(\Xi z_{i}\right)(t) \geq \frac{R_{1}}{R \bar{\ell}_{i}} \omega_{i}(t)
$$

where

$$
\begin{aligned}
\omega_{i}(t)= & \sum_{j=1}^{N}\left(\underline{\wp}_{i j}\left(\frac{R_{1}}{R}\right)^{\alpha_{i j}}+\underline{\omega}_{i j}\left(\frac{R_{1}}{R}\right)^{\beta_{i j}}+\left(\frac{R_{1}}{R}\right)^{\gamma_{i j}} \inf _{t \geq 0} \int_{-\theta_{i j}}^{0} \kappa_{i j}(t, u) d u\right) \\
& +\underline{q}_{i} \frac{\underline{v}_{i}\left(\frac{R_{1}}{R}\right)^{\alpha_{i i}}+\underline{r}_{i}}{\bar{k}_{i}}+\sum_{j=1, j \neq i}^{N} \underline{v}_{i j}\left(\frac{R_{1}}{R}\right)^{\alpha_{i i}}\left(\frac{R_{1}}{R}\right)^{\alpha_{i j}} .
\end{aligned}
$$

Since $\bar{\ell}_{i} \leq \underline{\wp}_{i i} R_{2}{ }^{\alpha i i}$, then

$$
\bar{\ell}_{i} \leq \underline{\wp}_{i i} R_{2}^{\alpha_{i i}} \leq \underline{\wp}_{i i} R_{2}^{\alpha_{i i}}+\omega_{i}(t),
$$

and

$$
1 \leq \frac{\wp_{i i} R_{2}^{\alpha_{i i}}}{\bar{\ell}_{i}} \leq \frac{\omega_{i}(t)}{\bar{\ell}_{i}}+\frac{\wp_{i i} R_{2}^{\alpha_{i i}}}{\bar{\ell}_{i}} \text {. }
$$

Thus

$$
\left(\Xi z_{i}\right)(t) \geq \frac{R_{1}}{R} \frac{\omega_{i}(t)}{\bar{\ell}_{i}} \geq \frac{R_{1}}{R} .
$$

Now, it must be proven that $\left(\Xi z_{i}\right)(t) \leq \frac{R_{2}}{R}$.

$$
\begin{aligned}
\left(\Xi z_{i}\right)(t) \leq & \frac{R_{2}}{R}\left[\sum_{j=1}^{N}\left(\bar{\wp}_{i j}\left(\frac{R_{2}}{R}\right)^{\alpha_{i j}}+\bar{\omega}_{i j}\left(\frac{R_{2}}{R}\right)^{\beta_{i j}}+\left(\frac{R_{2}}{R}\right)^{\gamma_{i j}} \sup _{t \geq 0} \int_{-\theta_{i j}}^{0} \kappa_{i j}(t, u)\right) d u\right. \\
& \left.+\bar{R}_{i} \frac{\bar{v}_{i}\left(\frac{R_{2}}{R}\right)^{\alpha_{i i}}+\bar{r}_{i}}{\underline{k}_{i}}+\sum_{j=1, j \neq i}^{N} \bar{v}_{i j}\left(\frac{R_{2}}{R}\right)^{\alpha_{i i}}\left(\frac{R_{2}}{R}\right)^{\alpha_{i j}}\right] .
\end{aligned}
$$


By hypothesis (A3), we get

$$
\sum_{j=1}^{N}\left(\bar{\wp}_{i j}\left(\frac{R_{2}}{R}\right)^{\alpha_{i j}}+\bar{\omega}_{i j}\left(\frac{R_{2}}{R}\right)^{\beta_{i j}}+\left(\frac{R_{2}}{R}\right)^{\gamma_{i j}} \sup _{t \geq 0} \int_{-\theta_{i j}}^{0} \kappa_{i j}(t, u) d u\right)+\bar{q}_{i} \frac{\bar{v}_{i}\left(\frac{R_{2}}{R}\right)^{\alpha_{i i}}+\bar{r}_{i}}{\underline{k}_{i}}+\sum_{j=1, j \neq i}^{N} \bar{v}_{i j}\left(\frac{R_{2}}{R}\right)^{\alpha_{i i}}\left(\frac{R_{2}}{R}\right)^{\alpha_{i j}} \leq \underline{\ell}_{i} .
$$

Let

$$
\Lambda=\sum_{j=1}^{N}\left(\bar{\wp}_{i j}\left(\frac{R_{2}}{R}\right)^{\alpha_{i j}}+\bar{\omega}_{i j}\left(\frac{R_{2}}{R}\right)^{\beta_{i j}}+\left(\frac{R_{2}}{R}\right)^{\gamma_{i j}} \sup _{t \geq 0} \int_{-\sigma_{i j}}^{0} \kappa_{i j}(t, u) d u\right)+\bar{q}_{i} \frac{\bar{v}_{i}\left(\frac{R_{2}}{R}\right)^{\alpha_{i i}}+\bar{r}_{i}}{\underline{k}_{i}}+\sum_{j=1, j \neq i}^{N} \bar{v}_{i j}\left(\frac{R_{2}}{R}\right)^{\alpha_{i i}}\left(\frac{R_{2}}{R}\right)^{\alpha_{i j}},
$$

then

$$
\frac{\ell_{i}}{\Lambda} \geq 1
$$

Consequently,

$$
\begin{aligned}
\left(\Xi z_{i}\right)(t) & \leq \frac{R_{2}}{R} \frac{\Lambda}{\underline{\ell}_{i}} \\
& \leq \frac{R_{2}}{R} .
\end{aligned}
$$

Then $\Xi$ is a mapping in $\Omega$ into itself.

Next, let us prove that $\Xi$ is completely continuous. Let $\Gamma(0, \xi)$ be a closed ball in the space $(B C(\mathbb{R}, \mathbb{R}))^{3}$. Let $V=\Xi(\Gamma(0, \xi))$ and $Y=\Xi Z$ for $Z \in \Gamma(0, \xi)$. It must be proven that $V$ is relatively compact.

Step1: $\{V(t), t \in \mathbb{R}\}$ represents a subset of $(B C(\mathbb{R}, \mathbb{R}))^{3}$ which is relatively compact.

- If $\xi \leq \frac{R_{2}}{R}$, then

$$
\begin{aligned}
|Y(t)|=|(\Xi Z)(t)| \leq & \frac{\sum_{j=1}^{N}\left(\bar{\wp}_{i j} \xi^{\alpha_{i j}}+\bar{\omega}_{i j} \xi^{\beta_{i j}}+\xi^{\gamma_{i j}} \sup _{t \geq 0} \int_{-\theta_{i j}}^{0} \kappa_{i j}(t, u) d u\right)+\bar{q}_{i} \frac{\bar{v}_{i} \xi^{\alpha_{i i}} \bar{\ell}_{i}}{\underline{\ell}_{i}}}{\underline{\ell}_{i}} \\
& +\frac{\sum_{j=1, j \neq i}^{N} \bar{v}_{i j} \xi^{\alpha_{i i}} \xi^{\alpha_{i j}}}{\underline{\ell}_{i}}=K
\end{aligned}
$$

which proves that $Y(t) \in \Gamma(0, K)$.

- If $\xi \geq \frac{R_{2}}{R}$, we have

$$
\begin{aligned}
|Y(t)| & =|(\Xi Z)(t)| \\
& \leq \frac{R_{2}}{R},
\end{aligned}
$$

and $Y(t) \in \Gamma\left(0, \frac{R_{2}}{R}\right)$.

Consequently, $\{V(t), t \in \mathbb{R}\}$ is relatively compact.

Step2: $V$ is equi-continuous. In fact, $t \rightarrow \ell_{i}(t), \digamma_{i}(t)$ are continuous, then for each $\varepsilon>0$, we can have $\delta>0$, in a certain way that for $|h|<\delta$, we get

$$
\begin{aligned}
\left|\ell_{i}(t+h)-\ell_{i}(t)\right| & \leq \frac{\varepsilon}{2\left\|\digamma_{i}\right\|}, \\
\left|\digamma_{i}(t+h)-\digamma_{i}(t)\right| & \leq \frac{\varepsilon}{2 \underline{\ell}_{i}}
\end{aligned}
$$


for all $i=1, \ldots, N$. Then

$$
\begin{aligned}
& |Y(t+h)-Y(t)|=\left|\int_{t+h}^{\infty} e^{-\int_{t+h}^{s} \ell_{i}(u) d u} \digamma_{i}(s) d s-\int_{t}^{\infty} e^{-\int_{t}^{s} \ell_{i}(u) d u} \digamma_{i}(s) d s\right| \\
& =\left|\int_{t}^{\infty} e^{-\int_{t}^{s} \ell_{i}(u+h) d u} \digamma_{i}(s+h) d s-\int_{t}^{\infty} e^{-\int_{t}^{s} \ell_{i}(u) d u} \digamma_{i}(s) d s\right| \\
& \leq\left|\int_{t}^{\infty} e^{-(s-t) \underline{\ell}_{i}}\right| \digamma_{i}(s+h)-\digamma_{i}(s) \mid d s \\
& +\left\|\digamma_{i}\right\|_{\infty} \int_{t}^{\infty}\left|\int_{t}^{s}\left[\ell_{i}(u+h)-\ell_{i}(u)\right] d u\right| \\
& e^{-\int_{t}^{s} \ell_{i}(u+h) d u+\theta\left(\int_{t}^{s} \ell_{i}(u) d u-\int_{t}^{s} \ell_{i}(u+h) d u\right)} d s \\
& \leq\left\|\digamma_{i}\right\|_{\infty} \int_{t}^{\infty}\left|\int_{t}^{s}\left[\ell_{i}(u+h)-\ell_{i}(u)\right] d u\right| \\
& e^{-(s-t) \underline{\ell}_{i}} e^{-\theta\left(\int_{t}^{s} \ell_{i}(u) d u-\int_{t}^{s} \ell_{i}(u+h) d u\right)} d s+\frac{\varepsilon}{2} \\
& \leq\left\|\digamma_{i}\right\|_{\infty} \int_{t}^{\infty} \int_{t}^{s}\left|\ell_{i}(u+h)-\ell_{i}(u)\right| d u e^{-(s-t) \underline{\ell}_{i}} d s+\frac{\varepsilon}{2} .
\end{aligned}
$$

By (9), we have

$$
\lim _{h \rightarrow 0} \int_{t}^{s}\left|\ell_{i}(u+h)-\ell_{i}(u)\right| d u=0 .
$$

Hence, using the Lebesgue dominated convergence theorem, we get

$$
\left\|\digamma_{i}\right\|_{\infty} \int_{t}^{\infty} \int_{t}^{s}\left|\ell_{i}(u+h)-\ell_{i}(u)\right| d u e^{-(s-t) \underline{\ell}_{i}} d s<\frac{\varepsilon}{2} .
$$

Hence,

$$
|Y(t+h)-Y(t)|<\varepsilon .
$$

Now, we have to prove that set $E=\left\{Y^{\rho}: Y^{\rho}=\rho \Xi Y^{\rho}, \rho \in(0,1)\right\}$ is bounded. In case that $Y^{\rho}$ the solution of $Y^{\rho}=\rho \Xi Y \rho$ for some $0<\rho<1$, then

$$
\begin{aligned}
\left|Y^{\rho}(t)\right| & \leq \rho\left|\Xi Y^{\rho}\right| \\
& <\left|\Xi Y^{\rho}\right| \\
& \leq \frac{R_{2}}{R} .
\end{aligned}
$$

Hence $\left\|Y^{\rho}\right\| \leq \frac{R_{2}}{R}$, it can be concluded that set $E=\left\{Y^{\rho}: Y^{\rho}=\rho \Xi Y^{\rho}, \rho \in(0,1)\right\}$ is bounded.

$\Xi$ a mapping of $\Omega$ into itself, and $\Xi: \Omega \rightarrow \Omega$ is completely continuous. Consequently, via Leray-Schauder fixed point theorem $\Xi$ has a fixed point $Z^{\star} \in \Omega$.

\section{Stabilities of the $(\mu, \eta)$-pseudo almost automorphic solutions}

\subsection{Asymptotic stability of the $(\mu, \eta)$-pseudo almost automorphic solutions}

Theorem 8. Under conditions (A1) - (A5), solution $Z^{\star}(t)$ given by theorem (7) is globally asymptotic stable. 
Proof. Let $Z(t)=\left(z_{1}(t), \ldots, z_{N}(t)\right)$ be another solution of system (5) and $\zeta=\left(\zeta_{1}, \ldots, \zeta_{N}\right)$ be a positive vector, such that $\iota=\min _{1 \leq i \leq N} \zeta_{i}$. The Lyapunov function is defined thusly

$$
V(t)=\sum_{i=1}^{N} \zeta_{i}\left|\ln z_{i}-\ln z_{i}^{*}\right| .
$$

The upper right derivative $D^{+} V($.$) of V($.$) given$

$$
\begin{aligned}
& D^{+} V(t) \leq-\sum_{i=1}^{N} \zeta_{i}\left[\sum _ { j = 1 } ^ { N } \left(\wp_{i j}(t)\left|\left(z_{j}\right)^{\alpha_{i j}}-\left(z_{j}^{\star}\right)^{\alpha_{i j}}\right|+q_{i}(t)\left[\left(\Psi z_{i}^{\star}\right)(t)-\left(\Psi z_{i}\right)(t)\right]\right.\right. \\
& +\omega_{i j}(t)\left|\left(z_{j}^{\star}\right)^{\beta_{i j}}\left(t-e_{i j}(t)\right)-z_{j}^{\beta_{i j}}\left(t-e_{i j}(t)\right)\right| \\
& \left.+\int_{-\theta_{i j}}^{0} \kappa_{i j}(t, u)\left|\left(z_{j}^{\star}\right)^{\gamma_{i j}}(u+t)-z_{j}^{\gamma_{i j}}(u+t)\right| d u\right) \\
& \left.+\sum_{j=1, j \neq i}^{N} v_{i j}(t)\left|\left(z_{i}^{\star}\right)^{\alpha_{i i}}(t)\left(z_{j}^{\star}\right)^{\alpha_{i j}}(t)-z_{i}^{\alpha_{i i}}(t) z_{j}^{\alpha_{i j}}(t)\right|\right] \\
& \leq-\sum_{i=1}^{N} \zeta_{i} \sum_{j=1}^{N} \wp_{i j}(t)\left|\left(z_{j}\right)^{\alpha_{i j}}-\left(z_{j}^{\star}\right)^{\alpha_{i j}}\right| \\
& \leq-\zeta_{i \underline{\wp}} \underline{i i}_{i}\left(z_{i}\right)^{\alpha_{i i}}(t)-\left(z_{i}^{*}\right)^{\alpha_{i i}}(t) \mid<0 .
\end{aligned}
$$

Consequently, solution $Z^{\star}(t)$ of system (5) is stable in Lyapunov sense. In fact, integrating (10) over $\left[T_{1}, t\right]$ leads to

$$
\int_{T_{1}}^{t} D^{+} V(s) d s \leq \int_{T_{1}}^{t}-\zeta_{i} \underline{\wp}_{i i}\left|\left(z_{i}\right)^{\alpha_{i i}}(s)-\left(z_{i}^{*}\right)^{\alpha_{i i}}(s)\right| d s,
$$

which gives

$$
\zeta_{i} \underline{\wp}_{i i} \int_{T_{1}}^{t}\left|\left(z_{i}\right)^{\alpha_{i i}}(s)-\left(z_{i}^{\star}\right)^{\alpha_{i i}}(s)\right| d s \leq V\left(T_{1}\right)-V(t),
$$

hence

$$
\int_{T_{1}}^{t}\left|\left(z_{i}\right)^{\alpha_{i i}}(s)-\left(z_{i}^{\star}\right)^{\alpha_{i i}}(s)\right| d s \leq \frac{V\left(T_{1}\right)}{\varliminf_{i i}} .
$$

Then, $\lim _{t \rightarrow \infty}\left|z_{i}^{\alpha_{i i}}(t)-\left(z_{i}^{\star}\right)^{\alpha_{i i}}(t)\right|=0$. This complete the proof.

\subsection{Exponential stability of the $(\mu, \eta)$-pseudo almost automorphic solutions}

Theorem 9. Under assumptions (A1)-(A5), assume that there exists constant $\lambda>0$ in a certain manner that, for all $1 \leq i \leq N$

$$
\lambda<\min _{i} \chi_{i i},
$$

where $\chi_{i}=\underline{\wp}_{i i} \frac{R_{1}}{R} \min \left(\alpha_{i i}\left(\frac{R_{1}}{R}\right)^{\alpha_{i i}-1}, \alpha_{i i}\left(\frac{R_{2}}{R}\right)^{\alpha_{i i}-1}\right)$. Then, $Z^{\star}(t)$ is globally exponential stable.

Proof. Let $Z(t)=\left(z_{1}(t), \ldots, z_{N}(t)\right)$ be another solution of system (5) and $\xi=\left(\xi_{1}, \ldots, \xi_{N}\right)$ be a positive vector. Let us define a Lyapunov function as follows

$$
V(t)=\sum_{i=1}^{N} \xi_{i} e^{\lambda t}\left|\ln z_{i}-\ln z_{i}^{\star}\right|
$$


Calculating an upper right $D^{+} V($.) derivative of $V($.$) , we obtain:$

$$
\begin{aligned}
D^{+} V(t) \leq & \sum_{i=1}^{N} \xi_{i}\left[\lambda e^{\lambda t}\left|\ln z_{i}-\ln z_{i}^{*}\right|-e^{\lambda t} \sum_{j=1}^{N}\left[\wp_{i j}(t)\left|\left(z_{j}\right)^{\alpha_{i j}}-\left(z_{j}^{*}\right)^{\alpha_{i j}}\right|\right.\right. \\
& +\omega_{i j}(t)\left|\left(z_{j}^{*}\right)^{\beta_{i j}}\left(t-e_{i j}(t)\right)-z_{j}^{\beta_{i j}}\left(t-e_{i j}(t)\right)\right| \\
& \left.+\int_{-\theta_{i j}}^{0} \kappa_{i j}(t, u)\left|\left(z_{j}^{\star}\right)^{\gamma_{i j}}(u+t)-z_{j}^{\gamma_{i j}}(u+t)\right| d u\right) \\
& +q_{i}(t)\left[\left(\Psi z_{i}^{*}\right)(t)-\left(\Psi z_{i}\right)(t)\right] \\
& \left.+\sum_{j=1, j \neq i}^{N} v_{i j}(t)\left|\left(z_{i}^{*}\right)^{\alpha_{i i}}(t)\left(z_{j}^{*}\right)^{\alpha_{i j}}(t)-z_{i}^{\alpha_{i i}}(t) z_{j}^{\alpha_{i j}}(t)\right|\right] \\
\leq & \left.\sum_{i=1}^{N} \xi_{i} e^{\lambda t}\left[\left|\ln z_{i}-\ln z_{i}^{*}\right|-e^{\lambda t} \sum_{j=1}^{N} \wp_{i j}(t) \mid\left(z_{j}\right)^{\alpha_{i j}}-\left(z_{j}^{\star}\right)^{\alpha_{i j}}\right]\right] \\
\leq & \sum_{i=1}^{N} \xi_{i} e^{\lambda t}\left[\lambda\left|\ln z_{i}-\ln z_{i}^{*}\right|-\wp_{i i}(t) \mid\left(z_{i}\right)^{\alpha_{i i}}-\left(z_{i}^{*}\right)^{\left.\alpha_{i i} \mid\right] .}\right.
\end{aligned}
$$

Since $Z(t), Z^{\star}(t)$ are solutions of system (5) in region $\Omega$, then $Z(t), Z^{\star}(t)$ satisfies

$$
\frac{R}{R_{2}}\left|z_{i}(t)-z_{i}^{\star}(t)\right| \leq\left|\ln z_{i}-\ln z_{i}^{*}\right| \leq \frac{R}{R_{1}}\left|z_{i}(t)-z_{i}^{\star}(t)\right| .
$$

Hence

$$
D^{+} V(t) \leq-\sum_{i=1}^{N} \xi_{i} e^{\lambda t} \frac{R}{R_{1}}\left|z_{i}-z_{i}^{\star}\right|\left[-\lambda+\min _{i} \chi_{i}\right]<0 .
$$

Consequently, solution $Z^{\star}(t)$ of system (5) can be considered stable in Lyapunov sense. Furthermore,

$$
\sum_{i=1}^{N} \xi_{i} e^{\lambda t}\left|\ln z_{i}-\ln z_{i}^{\star}\right| \leq V(0),
$$

and

$$
\sum_{i=1}^{N} e^{\lambda t} \frac{R}{R_{2}}\left|z_{i}(t)-z_{i}^{\star}(t)\right| \leq \frac{V(0)}{\min _{i} \xi_{i}},
$$

in addition

$$
\sum_{i=1}^{N}\left|z_{i}(t)-z_{i}^{*}(t)\right| \leq \frac{R_{2}}{R} e^{-\lambda t} \frac{V(0)}{\min _{i} \xi_{i}},
$$

Therefore, solution $Z^{\star}(t)$ of system (5) can be global exponential stable.

\section{Numerical example and simulation}

We present in this section an example to show our findings feasibility. 


\subsection{Example}

Let consider the following model:

$$
\left\{\begin{array}{l}
z_{i}^{\prime}(t)=z_{i}(t)\left[\ell_{i}(t)-\sum_{j=1}^{2} \wp_{i j}(t) z_{j}^{\alpha_{i j}}(t)-\sum_{j=1}^{2} \omega_{i j}(t) z_{j}^{\beta_{i j}}\left(t-e_{i j}(t)\right)\right. \\
\left.-q_{i}(t) p_{i}(t)-\sum_{j=1, j \neq i}^{2} v_{i j}(t) z_{i}^{\alpha_{i i}}(t) z_{j}^{\alpha_{i j}}(t)-\sum_{j=1}^{2} \int_{-\theta_{i j}}^{0} \kappa_{i j}(t, s) z_{j}^{\gamma_{i j}}(t+s) d s\right], \\
p_{i}^{\prime}(t)=r_{i}(t)-k_{i}(t) p_{i}(t)+v_{i}(t) z_{i}^{\alpha_{i i}}(t) .
\end{array}\right.
$$

Now, let consider a measure $\mu$ in the event that its Radon-Nikodym derivative is

$$
\rho_{1}(t)=e^{\sin (t)}, t \in \mathbb{R} .
$$

and a measure $\eta$ in the event that its Radon-Nikodym derivative is

$$
\rho_{2}(t)=\left\{\begin{array}{l}
e^{t} \text { if } t \leq 0 \\
1 \text { ift }>0
\end{array}\right.
$$

Since

$$
\limsup _{s \rightarrow \infty} \frac{\mu([-s, s])}{\eta([-s, s])}=\limsup _{r \rightarrow \infty} \frac{\int_{-s}^{s} \rho_{1}(t) d t}{\int_{-s}^{s} \rho_{2}(t) d t}<\infty .
$$

Then $\mu, \eta$ satisfies (A1). In addition, for

$$
\begin{gathered}
\left(\omega_{i j}(t)\right)_{1 \leq i, j \leq 2}=\left(\begin{array}{cc}
\frac{1}{4} & 0 \\
0 & \frac{1}{7}
\end{array}\right),\left(v_{i j}(t)\right)_{1 \leq i, j \leq 2, i \neq j}=\left(\begin{array}{l}
0 \\
0
\end{array}\right) \\
\ell=\left(\ell_{i}(t)\right)_{1 \leq i \leq 2}\left(\begin{array}{l}
17+\sin \left(\frac{2}{2+\sin (t)+\cos (t)}\right)^{2} \\
16+\sin \left(\frac{2}{2+\sin (t)+\cos (t)}\right)^{2}
\end{array}\right)
\end{gathered}
$$

and

$$
\begin{aligned}
& q=\left(q_{i}\right)_{1 \leq i \leq 2}=\left(\begin{array}{c}
\frac{1}{3} \cos \left(\frac{\sqrt{2} t}{2+\sin (t)+\cos (t)}\right)^{2}+\frac{1}{8} e^{-t^{2}} \\
\frac{1}{6}+\frac{1}{7} \sin ^{2}\left(\frac{\sqrt{2} t}{2+\sin (t)+\cos (t)}\right)
\end{array}\right), \\
& r=\left(r_{i}\right)_{1 \leq i \leq 2}=\left(\begin{array}{c}
\frac{1}{8}+\sin \left(\frac{\sqrt{3} t}{2+\sin (t)+\cos (t)}\right)^{2}+\frac{1}{8} e^{-t^{2}} \\
\frac{1}{9}+\cos \left(\frac{\sqrt{2} t}{2+\sin (t)+\cos (t)}\right)^{2}+\frac{1}{9} e^{-|t|}
\end{array}\right), \\
& k=\left(k_{i}\right)_{1 \leq i \leq 2}=\left(\begin{array}{c}
\frac{1}{8}\left(1+\frac{1}{9} \cos \left(\frac{\sqrt{2} t}{2+\sin (t)+\cos (t)}\right)\right) \\
\frac{1}{9}\left(1+\frac{1}{4} \sin \left(\frac{\sqrt{2} t}{2+\sin (t)+\cos (t)}\right)\right.
\end{array}\right), \\
& v=\left(v_{i}\right)_{1 \leq i \leq 2}=\left(\begin{array}{c}
\frac{1}{8}+\left(\cos \left(\frac{\sqrt{3} t}{2+\sin (t)+\cos (t)}\right)\right)^{2}+\frac{1}{8} e^{-t} \\
\frac{1}{9} \sin ^{2}\left(\frac{\sqrt{2} t}{2+\sin (t)+\cos (t)}\right)+\frac{1}{9} e^{-t^{2}}
\end{array}\right), \\
& \left(\wp_{i j}(t)\right)_{1 \leq i, j \leq 2}=\left(\begin{array}{cc}
3+\sin \left(\frac{\sqrt{2} t}{2+\sin (t)+\cos (t)}\right)^{2}+\exp \left(-t^{2}\right) & 0 \\
0 & +\cos \left(\frac{\sqrt{2} t}{2+\sin (t)+\cos (t)}\right)^{2}+\exp \left(-t^{2}\right)
\end{array}\right),
\end{aligned}
$$

$\kappa_{11}(t, s)=\frac{1}{2}, \kappa_{22}(t, s)=\frac{3}{2}$. They delay as follows $e_{12}=0.1, e_{21}=0.3, \theta_{11}=0.2, \theta_{22}=0.4$, and $\alpha_{11}=$ 2, $\alpha_{22}=\frac{3}{2}, \alpha_{21}=1, \alpha_{12}=0, \beta_{22}=\frac{3}{2}, \beta_{12}=1, \beta_{21}=1, \gamma_{11}=2, \gamma_{22}=\frac{3}{2}$. all the conditions (A1)-(A5) are satisfied.

According to Theorems (7), (8) and (9), system (12) has a fixed ( $\mu, \eta)$-pseudo almost automorphic solution. This latter can be globally asymptotic and exponential stable. 


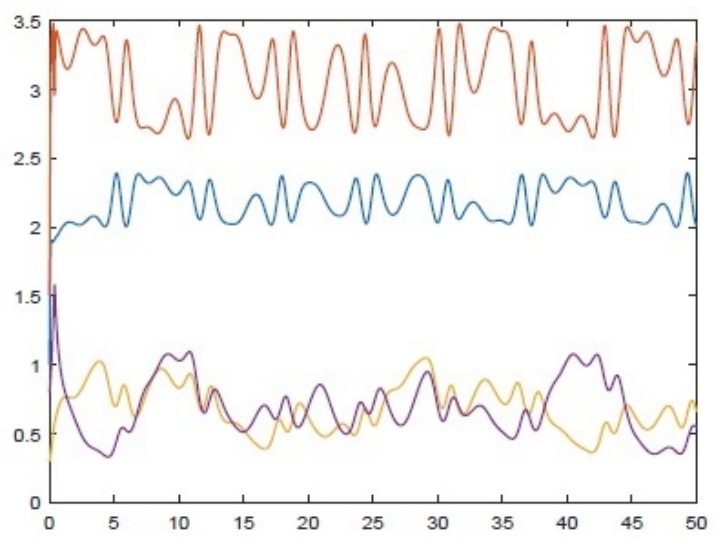

Figure 1: Curve of $(\mu, \eta)$-pseudo almost automorphic solution $\left(z_{1}(t), z_{2}(t), p_{1}(t), p_{2}(t)\right)$ of equation (12) for initial value $(1,1.5,0.2,0.8)$

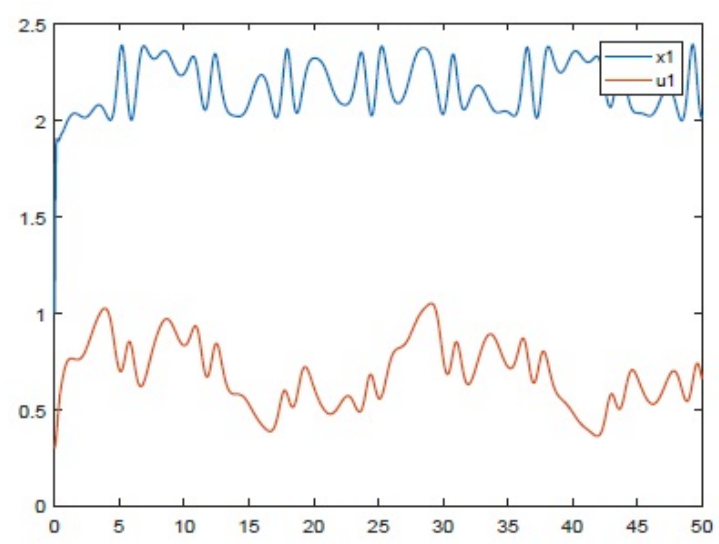

Figure 2: Curve of $(\mu, \eta)$-pseudo almost automorphic solution $\left(z_{1}(t), p_{1}(t)\right)$ of equation (12) for initial value $(1,0.8)$

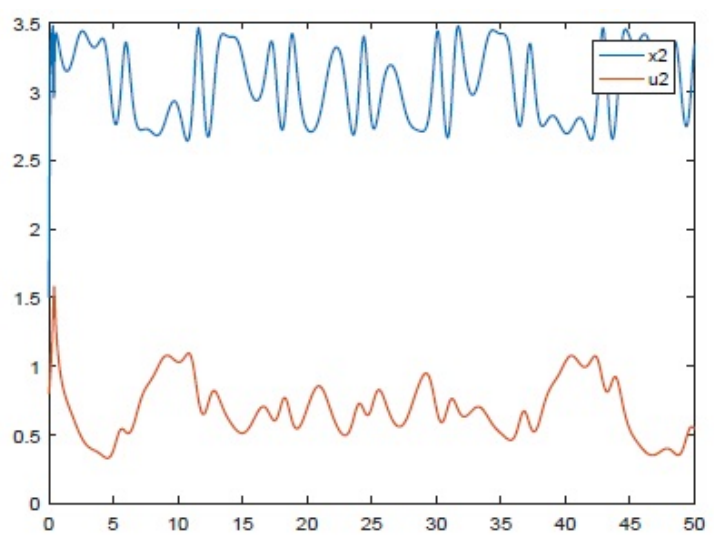

Figure 3: Curve of $(\mu, \eta)$-pseudo almost automorphic solution $\left(z_{2}(t), p_{2}(t)\right)$ of equation (12) for initial value $(1.5,0.8)$ 


\section{Conclusion}

The $(\mu, \eta)$-pseudo almost automorphic functions consist of a bigger class of functions, so a highly complex behavior can be expressed for these functions. Within this work, a Lotka-Volterra $N$-species Gilpin-Ayala type competitive model with feedback control has been investigated. Applying the Leray-Schauder Alternative theorem and the exponential dichotomy, we obtain new adequate conditions for the existence, permanence and globally exponential stability and asymptotic stability of $(\mu, \eta)$-pseudo almost automorphic solutions. After that, an example has been provided to show the effectiveness of the achieved results. As a matter of fact, we can apply the method of this paper with the purpose of studying other mathematical, economical and biological models.

Acknowledgement: The authors would like to thank the anonymous reviewer and the editor for their constructive comments, which greatly improved the quality of this paper.

\section{References}

[1] Amdouni. M, Chérif. F, The pseudo almost periodic solutions of the new class of Lotka-Volterra recurrent neural Networks with mixed delays, Chaos, Solitons and Fractals. 113, 79-88, 2018.

[2] Amdouni.M, Chérif.F, Pseudo Almost Periodic Solutions of the Third Order Differential Equation with Continuous Delay. Appl. Math. Inf. Sci. 14( 2), 1-14, 2020.

[3] Ammar.B, Chérif.F, M.Alimi.A, Existence and uniqueness of pseudo almost periodic solutions of reccurent neural networks with time varying coefficients and mixed delays, IEE trans.neural netw. 23, 2012.

[4] Ahmad.S, On nonautonomous Lotka-Volterra competition equations, Proc. Amer. Math. Soc. 177, 199-204, 1993.

[5] Ayala.F.J, Gilpin.M.E, Ehrenfeld. J.G,Competition between species: theoretical models and experimental tests, Theoretical Population Biology. 4, 331-356, 1973.

[6] Blot.J, Cieutat.P, M. N'Guérékata.G, Superposition operators between various almost periodic function spaces and applications, Communications in Mathematical Analysis. 6, 42-70, 2009.

[7] Blot.J, Mophou.G.M, M. N’Guérékata.G, Pennequin.D, Weighted pseudo almost automorphic functions and applications to abstract differential equations. Nonlinear Analysis. 71, 903-909, 2009.

[8] Blot.J, Cieutat.P, Ezzinbi.K, Measure theory and pseudo almost automorphic functions: new developments and applications. Nonlinear Anal. 75, 2426-2447, 2012.

[9] Cieutat.P, F.Samir, M.N'Guérékata.G, Composition of pseudo almost periodic and pseudo almost automorphic functions and applications to evolution equations, Applicable Analysis. 89, 11-27, 2010.

[10] Chattopadhyay.J, Effect of toxic substance on a two-species competitive system, Ecol. Model. 84, 287-289, 1996.

[11] Chen.F, Average conditions for permanence and extinction in nonautonomous Gilpin-Ayala competition model, Nonlinear Analysis: Real World Applications. 4, 885-915, 2006.

[12] Chen.F, Some new results on the permanence and extinction of nonautonomous Gilpin-Ayala type competition model with delays, Nonlinear Analysis: Real World Applications. 7, 1205-1222, 2006.

[13] Diagana. T, Ezzinbi. K, Miraoui. M, Pseudo almost periodic and pseudo almost automorphic solutions to some evolution equations involving theoretical measure theory. Cubo. 16(2), 1-31, 2014.

[14] Fan.M, Wang.K, Global periodic solutions of a generalized n-species Gilpin-Ayala competition model, Comput.Math. Appl. 40, 1141-115, 2000.

[15] Fan.M, Wang.K, Jiang.D, Existence and global attractivity of positive periodic solutions of periodic n-species Lotka-Volterra competition systems with several deviating arguments, Mathematical Biosciences. 160, 47-61, 1999.

[16] Geng.J, Xia.Y, Almost periodic solutions of a nonlinear ecological model, Commun Nonlinear Sci Numer Simulat. 16, 25752597, 2011.

[17] Granas.A, Dugundji.J, Fixed Point Theory, Springer-Verlag ( New York,2003).

[18] Gopalsamy.K, Weng.P.X, Feedback regulation of logistic growth, Inernat.J.Math. Sci. 16, 177-192, 1992.

[19] Gopalsamy.K,Stability and Oscillations in Delay Differential Equations of Population Dynamics. Kluwer Academic Publishers( London, 1992).

[20] Gilpin. M.E, Ayala.J.F, Global models of growth and competition, Proc. Natl. Acad. Sci. USA. 70, 3590-3593, 1973.

[21] Liu.G, Yan.J, Positive Periodic Solutions for a Neutral Differential System with Feedback Control, Computers and Mathematics with Applications. 52, 401-410, 2006.

[22] Muhammadhaji.A, Teng.Z, Positive periodic solutions of n-species Lotka-Volterra cooperative systems with delays. Vietnam Journal of Mathematics. 40, 453-467, 2012. 
[23] Toka.D, Almost Automorphic Type and Almost Periodic Type Functions in Abstract Spaces (Springer International Publishing Switzerland,2013).

[24] Teng.Z, Nonautonomous Lotka-Volterra Systems with Delays, Journal of Differential Equations. 179, 538-561, 2002.

[25] Xia.Y, Han.M, Kun Huang.K, Global attractivity of an almost periodic $N$-species nonlinear ecological competitive model,J.Math.Anal.Appl. 337, 144-168, 2008.

[26] Xia.Y, Cao.J, Zhang.H, Chen.F, Almost periodic solutions of $n$-species competitive system with feedback controls,J. Math. Anal. Appl. 294, 503-522, 2004.

[27] Xiao.Y, Tang.S, Chen.J, Permanence and periodic solution in competitive system with feedback controls, Math. Comput. Modelling. 27, 33-37, 1998.

[28] Yongkun.L, Periodic solutions for delay Lotka-Volterra competition systems, Journal of Mathematical Analysis and Applications 246, 230-244, 2000.

[29] Zhao.X.Q, The qualitative analysis of $n$-species Lotka-volterra periodic competition systems, Mathl. Comput. Modelling. 15, 3-8, 1991.

[30] Zhou.T, Wang.M, Li.C, Almost periodic solution for multidirectional associative memory neural network with distributed delays, Math Comput Simul. 107, 52-60, 2015.

[31] Zhao.K, Ye.Y, Four positive periodic solutions to a periodic Lotka-Volterra predatory-prey system with harvesting terms. Nonlinear Analysis. Real World Applications. 4, 2448-2455, 2010. 